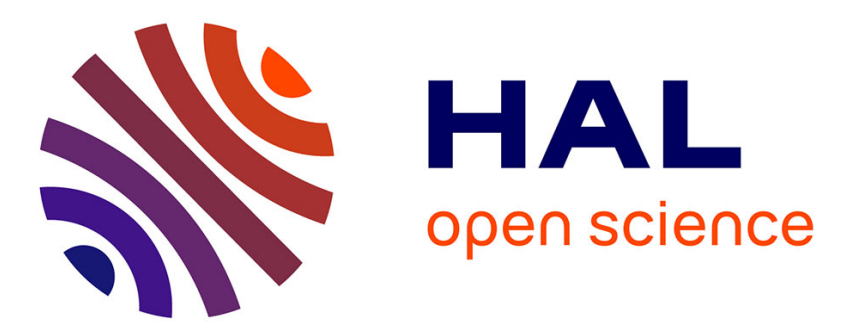

\title{
Simulation of crashworthiness problems with improved contact algorithms for implicit time integration
}

\author{
Ludovic Noels, Laurent Stainier, Jean-Philippe Ponthot
}

\section{To cite this version:}

Ludovic Noels, Laurent Stainier, Jean-Philippe Ponthot. Simulation of crashworthiness problems with improved contact algorithms for implicit time integration. International Journal of Impact Engineering, 2006, 32 (5), pp.799-825. 10.1016/j.ijimpeng.2005.04.010 . hal-01007298

\section{HAL Id: hal-01007298 \\ https://hal.science/hal-01007298}

Submitted on 18 Nov 2021

HAL is a multi-disciplinary open access archive for the deposit and dissemination of scientific research documents, whether they are published or not. The documents may come from teaching and research institutions in France or abroad, or from public or private research centers.
L'archive ouverte pluridisciplinaire HAL, est destinée au dépôt et à la diffusion de documents scientifiques de niveau recherche, publiés ou non, émanant des établissements d'enseignement et de recherche français ou étrangers, des laboratoires publics ou privés.

\section{다)(1) $(5$}

Distributed under a Creative Commons Attribution - NonCommercial| 4.0 International 


\title{
Simulation of crashworthiness problems with improved contact algorithms for implicit time integration
}

\author{
L. Noels ${ }^{1}$, L. Stainier ${ }^{2}$, J.-P. Ponthot* \\ University of Liège, LTAS-Milieux Continus and Thermomécanique, Chemin des Chevreuils 1, B-4000 Liège, Belgium
}

When studying crashworthiness problems, contact simulation can be the source of a number of problems. A first one is the discontinuities in the normal evolution for a boundary discretized by finite elements. Another problem is the treatment of the contact forces that can introduce numerical energy in the system. In this paper, we propose to combine a method of discontinuity smoothing with the energy-momentum consistent scheme that recently appeared in the literature.

Keywords: Energy conserving; Momentum conserving; Dynamics; Contact; Finite-elements method

\section{Introduction}

When studying impact problems, time integration of the equations of evolution occurs in the non-linear range. An important source of non-linearity results from the bodies contact interactions. Treatment of the contact can be achieved with a penalty method, with an augmented Lagrangian method or with a Lagrangian method $[1,2]$. For each method, two problems must be carefully taken into account: the first one results from the finite-elements discretization that leads

\footnotetext{
${ }^{*}$ Corresponding author. Tel.: + 3243669310 ; fax: + 3243669141.

E-mail address: jp.ponthot@ulg.ac.be (J.-P. Ponthot).

${ }^{1}$ Research Fellow at the Belgian National Fund for Scientific Research (FNRS).

${ }^{2}$ Research Associate at the Belgian National Fund for Scientific Research (FNRS).
} 
to discontinuous normal evolution, and the second one results from the fact that a non-linearity can lead to instabilities of the time integration algorithm.

The first problem can be resolved with some numerical techniques such as gap smoothing based on a signed function as proposed by Belytschko et al. [3]. Another technique is to consider a segment-to-segment approach (and not a node-on-segment approach) as proposed by Puso and Laursen [4]. Nevertheless, Graillet and Ponthot [5] proposed a node-on-segment approach that consists in adapting the normal of the surface, leading to a continuous discretization. This method has the advantage of being easy to implement and to lead to an efficient algorithm.

The second problem is solved thanks to the recent development of the energy momentum conserving algorithm (EMCA) initially proposed by Simo and Tarnow [6]. It consists in a midpoint scheme with an adequate evaluation of the forces leading to a stable algorithm in the nonlinear range. This adequate evaluation of the contact forces was given for a penalty method by Armero and Petöcz [7,8] to simulate frictional and frictionless contact. This method leads to penetration of the surface (as in a classical penalty method) and therefore to an accumulation of energy during the contact interaction. But the work of the contact forces is equal to the dissipation resulting from the friction after the contact is released. Laursen and Chawla $[9,10]$ have developed penalty and augmented Lagrangian methods leading to unconditional dissipation of the energy. Recently, Laursen and Love $[11,12]$ have extended these methods to geometric admissibility by the use of velocity corrections.

In this paper, we propose to rewrite the contact formulation proposed by Armero and Petöcz [7,8], taking into account the adaptation of the normal that leads to a continuous normal evolution. Moreover, the contact formulation is rewritten to be able to consider a 3-dimensionalboundary composed of Coons patches. To achieve this goal, the formulations that depend on the curvilinear coordinates have been rewritten in such a way that they depend only on the nodes projections and no more on the coordinates. This method allows one to consider frictional forces when the slave node moves from one patch to another.

The goal of this paper is to present a robust and accurate method that leads to an implicit time integration algorithm able to deal with crashworthiness problems. This kind of problems is generally solved with an explicit algorithm. Nevertheless, we think that such problems can be more accurately solved with an implicit scheme without loss of robustness or efficiency [13]. Moreover we are interested in combining both the implicit and explicit scheme while dealing with time integration. Such a combination can reduce the computational time without loss of accuracy [13-15]. Nevertheless it requires an efficient implicit time integration method. The purpose of this paper is to study the treatment of the contact interactions to provide such a robust time integration algorithm. In this paper we will study crashworthiness problems with an implicit method to demonstrate the robustness of the contact treatment in this method.

In Section 2 we will present some preliminaries on continuous dynamics, finite-elements discretization and time integration. Next, in Section 3, we will present the classical contact formulation and the method proposed by Graillet in order to get a continuous normal. Adaptation of the Armero and Petöcz [7,8] formulation, with the continuous normal method, that will lead to verify the conservation laws will be presented in Section 4. Finally, in Section 5, numerical examples will be presented to illustrate the efficiency, the robustness and the accuracy of the method. 


\section{Preliminaries}

After having briefly recalled the notations used in this paper, we will establish the continuous dynamics conservation laws. Next, we will introduce a finite-elements discretization and the EMCA. Readers interested in the basics of time integration algorithms can refer to classical books $[16,17]$.

\subsection{Notations}

Let $\mathbb{V}^{1}, \mathbb{V}^{2} \subset \mathbb{R}^{3}$ be the manifold of the points defining two bodies and $\mathbb{S}^{1}, \mathbb{S}^{2} \subset \mathbb{R}^{3}$ be the manifold of their respective boundary (Fig. 1). Two configurations are under consideration: the initial configuration that is referred to with a subscript 0 , and the current configuration at time $t$. Each boundary $\mathbb{S}^{i}$ is decomposed into two parts: the first one, $\mathbb{S}_{\vec{x}}^{i}$, is the part where the displacements are known and the second one, $\mathbb{S}_{\vec{T}}^{i}$, is the part where the traction is imposed. Let $\vec{x}$ be the current positions and $\vec{x}_{0}$ be the initial positions. Therefore, the two-point gradient of deformation tensor is defined by

$$
\mathbf{F} \equiv \frac{\partial \vec{x}}{\partial \vec{x}_{0}} \quad \text { with } \mathbf{f} \equiv \mathbf{F}^{-1} \text { and } J \equiv \operatorname{det} \mathbf{F} .
$$

Let $\rho_{0}: \mathbb{V}_{0} \rightarrow \mathbb{R}_{+}$be the initial density. Let $\mathbb{X}^{i}$ be the manifold of the admissible positions for the body $i$ :

$$
\mathbb{X}^{i} \equiv\left\{\vec{x}: \mathbb{V}_{0}^{i} \rightarrow \mathbb{R}^{3} \mid\left[J>0 \text { and }\left.\vec{x}\right|_{\mathbb{S}_{\vec{x}}^{i}}=\overline{\bar{x}}\right] \forall \vec{x}_{0} \in \mathbb{V}_{0}^{i}\right\}
$$

with $\overline{\vec{x}}$ the known positions. Let $t$ be the current time and let $\mathbb{T}=\left[0, t_{f}\right]$ be the integration interval. Therefore, the motion of the body is defined by $\forall i=1,2: t \in \mathbb{T} \rightarrow \vec{x}(t) \in \mathbb{X}^{i}$. During this motion, the body is subject to specific load $\vec{b}(t): \mathbb{V}_{0}^{i} \times \mathbb{T} \rightarrow \mathbb{R}^{3}$. In this paper, we assume this load is equal to zero. Let $\boldsymbol{\Sigma}$ be the Cauchy stress tensor. For each body $i$, boundary pressures $\vec{T}_{S^{i}}(t)$ : $\mathbb{S}_{\vec{T} 0}^{i} \times \mathbb{T} \rightarrow \mathbb{R}^{3}$ lead to the condition $\vec{T}_{S^{i}}(t)=\Sigma(t) \vec{n}(t)$ with $\vec{n}$ the outward unit normal to $\mathbb{S}^{i}$. Let us assume that the external forces result only from the contact interaction. Therefore, it leads from

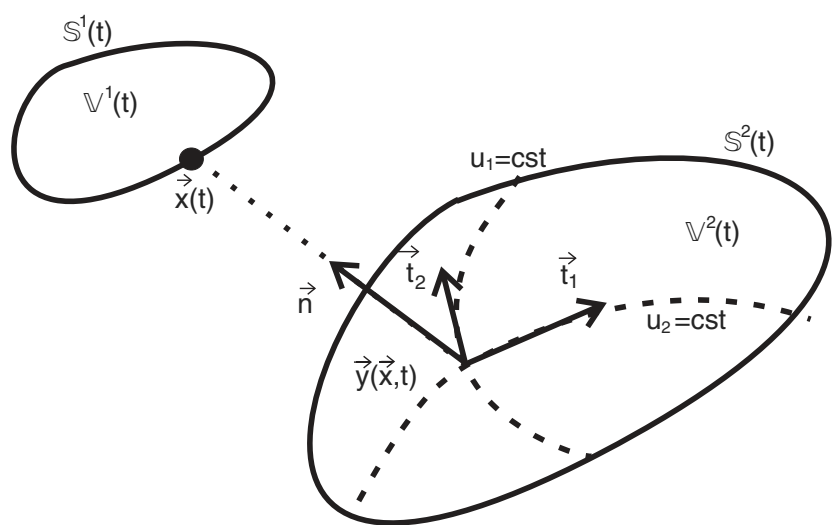

Fig. 1. Definition of the bodies interaction. 
the geometrical admissibility and from the action-reaction principle to

$$
\mathbb{S}_{\vec{T}}^{1}(t)=\mathbb{S}_{\vec{T}}^{2}(t) \quad \text { and } \quad \vec{T}_{S^{1}}\left(\vec{x}_{0}, t\right)=-\vec{T}_{S^{2}}\left(\vec{x}_{0}, t\right) \quad \forall \vec{x}_{0} \in \mathbb{S}_{\vec{T}}^{1} .
$$

The body is now decomposed into finite elements thanks to shape functions $\varphi^{\xi_{i}}: \mathbb{V}_{0}^{i} \rightarrow \mathbb{R}$ with $\xi_{i} \in\left[1, N_{i}\right]\left(N_{i}\right.$ the total number of nodes of body $\left.i=1,2\right)$, and with $\varphi^{\xi_{i}}\left(\vec{x}_{0}^{\mu_{j}}\right)=\delta_{\xi_{i}}^{\mu_{j}}(\delta$ is the Kronecker symbol). It leads for each node $\xi_{i} \in\left[1, N_{i}\right]$ and for each initial position $\vec{x}_{0} \in \mathbb{V}_{0}^{i}$ to

$$
\vec{x}\left(\vec{x}_{0}\right)=\varphi^{\xi_{i}}\left(\vec{x}_{0}\right) \vec{x}^{\xi_{i}}, \quad \dot{\vec{x}}\left(\vec{x}_{0}\right)=\varphi^{\xi_{i}}\left(\vec{x}_{0}\right) \dot{\vec{x}}^{\xi_{i}} \quad \text { and } \quad \ddot{\vec{x}}\left(\vec{x}_{0}\right)=\varphi^{\xi_{i}}\left(\vec{x}_{0}\right) \ddot{\vec{x}}^{\xi_{i}},
$$

where Einstein's notations are used. Let $\vec{v}$ be an admissible virtual displacement defined by the manifold for body $i$ :

$$
\mathbb{D}^{i} \equiv\left\{\vec{v}: \mathbb{V}_{0}^{i} \rightarrow \mathbb{R}^{3} \mid\left[\left.\vec{v}\right|_{\mathbb{S}_{\vec{x}}^{i}}=0, \vec{v}\left(\vec{x}_{0}, 0\right)=0, \vec{v}\left(\vec{x}_{0}, t_{f}\right)=0 \forall \vec{x}_{0} \in \mathbb{V}_{0}^{i}\right]\right\} .
$$

Let $\mathbb{F}^{i} \subset \mathbb{D}^{i}$ be the manifold of admissible virtual displacements $\delta \vec{x}$ that can be decomposed such as (4).

\subsection{Continuum dynamics}

The following quasi-variational principle (principle of virtual work of forces) must hold $\forall \delta \vec{x} \in \mathbb{F}^{i}[18$, p. 412]:

$$
\int_{0}^{t_{f}} \sum_{i=1,2}\left\{\int_{\mathbb{V}^{i}}\left[\rho \ddot{\vec{x}} \cdot \delta \vec{x}+\Sigma^{\mathrm{T}}: \frac{\partial \delta \vec{x}}{\partial \vec{x}}\right] \mathrm{d} \mathbb{V}^{i}-\int_{\mathbb{S}_{\vec{T}}^{i}}\left[\vec{T}_{S^{i}} \cdot \delta \vec{x}\right] \mathrm{d} \mathbb{S}^{i}\right\} \mathrm{d} t=0 .
$$

Integrating by parts, one gets

$$
\underbrace{\sum_{i=1,2} \int_{\mathbb{V}^{i}}\{\rho \ddot{\vec{x}} \cdot \delta \vec{x}\} \mathrm{d} \mathbb{V}^{i}}_{\equiv \delta K}=\underbrace{\sum_{i=1,2} \int_{\mathbb{S}_{\vec{T}}^{i}}\left\{\vec{T}_{S^{i}} \cdot \delta \vec{x}\right\} \mathrm{d} \mathbb{S}^{i}}_{\equiv \delta W_{\text {cont }}}-\underbrace{\sum_{i=1,2} \int_{\mathbb{V}^{i}}\left\{\boldsymbol{\Sigma}^{\mathrm{T}}: \frac{\partial \delta \vec{x}}{\partial \vec{x}}\right\} \mathrm{d} \mathbb{V}^{i}}_{\equiv \delta W_{\text {int }}} \forall t \in \mathbb{T}
$$

with $\delta W_{\text {int }}, \delta W_{\text {cont }}$ and $\delta K$ respectively the virtual work of internal forces, the virtual work of contact forces and the virtual work of inertia forces. This principle leads to the dynamics conservation laws.

\subsubsection{Conservation of linear momentum}

Let $\vec{L}$ be the linear momentum defined by

$$
\vec{L} \equiv \sum_{i=1,2} \int_{\mathbb{V}^{i}}\{\rho \dot{\vec{x}}\} \mathrm{d} \mathbb{V}^{i}=\sum_{i=1,2} \int_{\mathbb{V}_{0}^{i}}\left\{\rho_{0} \dot{\vec{x}}\right\} \mathrm{d} \mathbb{V}_{0}^{i},
$$

where the conservation of mass $\left(\rho \mathrm{d} \mathbb{V}=\rho_{0} d \mathbb{V}_{0}\right)$ is used. Using Eq. (3) and assuming pure Neumann boundary conditions (i.e. $\mathbb{S}_{\vec{x}}=\emptyset$ ), if $\delta \vec{x} \in \mathbb{F}^{i}$ is taken constant, Eq. (7) leads to the conservation of linear momentum

$$
\dot{\vec{L}}=\int_{\mathbb{S}_{\vec{T}}^{1}}\left\{\vec{T}_{S^{1}}-\vec{T}_{S^{2}}\right\} \mathrm{d} \mathbb{S}^{1}=0 \quad \forall t \in \mathbb{T} .
$$




\subsubsection{Conservation of angular momentum}

Let $\vec{J}$ be the angular momentum defined by

$$
\vec{J} \equiv \sum_{i=1,2} \int_{\mathbb{V}^{i}}\{\rho \vec{x} \wedge \dot{\vec{x}}\} \mathrm{d} \mathbb{V}^{i}=\sum_{i=1,2} \int_{\mathbb{V}_{0}^{i}}\left\{\rho_{0} \vec{x} \wedge \dot{\vec{x}}\right\} \mathrm{d} \mathbb{V}_{0}^{i}
$$

Using Eq. (3), assuming pure Neumann boundary conditions (i.e. $\mathbb{S}_{\vec{x}}=\emptyset$ ), and taking $\delta \vec{x}=\vec{\eta} \wedge \vec{x}$ with $\vec{\eta}$ constant, since $\boldsymbol{\Sigma}$ is symmetric, since $\vec{\eta}$ is an arbitrary constant, and since the points of the two surfaces have the same position $\vec{x}$, Eq. (7) leads to the conservation of angular momentum

$$
\dot{\vec{J}}=\int_{\mathbb{S}_{\vec{T}}^{1}}\left\{\vec{x} \wedge\left[\vec{T}_{S^{1}}-\vec{T}_{S^{2}}\right]\right\} \mathrm{d} \mathbb{S}^{1}=0 \quad \forall t \in \mathbb{T} .
$$

\subsubsection{Conservation of energy}

Let $K, W_{\text {int }}$ and $W_{\text {cont }}$ respectively be the kinetic energy, the internal forces work and the contact forces work, with

$$
\begin{aligned}
& K \equiv \sum_{i=1,2} \int_{\mathbb{V}^{i}}\left\{\frac{1}{2} \rho \dot{\vec{x}}^{2}\right\} \mathrm{d} \mathbb{V}^{i}=\sum_{i=1,2} \int_{\mathbb{V}_{0}^{i}}\left\{\frac{1}{2} \rho_{0} \dot{\vec{x}}^{2}\right\} \mathrm{d} \mathbb{V}_{0}^{i}, \\
& \dot{W}_{\text {int }} \equiv \sum_{i=1,2} \int_{\mathbb{V}^{i}}\left\{\Sigma^{\mathrm{T}}:[\dot{\mathbf{F}} \mathbf{f}]\right\} \mathrm{d} \mathbb{V}^{i}, \\
& \dot{W}_{\text {cont }} \equiv \sum_{i=1,2} \int_{\mathbb{S}_{\vec{T}}^{i}}\left\{\vec{T}_{S^{i}} \cdot \dot{\vec{x}}\right\} \mathrm{d} \mathbb{S}^{i},
\end{aligned}
$$

where the conservation of mass is used. Let us note that $\dot{W}_{\text {cont }} \neq 0$ since the points on the two surfaces do not necessarily have the same velocity. Let us split the contact forces $\vec{T}_{S^{i}}$ into a normal force $t_{N} \vec{n}$ (with $t_{N}: \mathbb{S}_{0}^{i} \times \mathbb{T} \rightarrow \mathbb{R}^{+}$the pressure) and a friction force $\vec{T}$. Since the normal velocities are the same for the two surfaces, it yields

$$
\dot{W}_{\mathrm{cont}}=\underbrace{\sum_{i=1,2} \int_{\mathbb{S}_{\vec{T}}^{i}}\left\{t_{N} \vec{n} \cdot \dot{\vec{x}}\right\} \mathrm{d} \mathbb{S}^{i}}_{=0}+\underbrace{\sum_{i=1,2} \int_{\mathbb{S}_{\vec{T}}^{i}}\{\vec{T} \cdot \dot{\vec{x}}\} \mathrm{d} \mathbb{S}^{i}}_{\equiv-\dot{\Delta}_{\mathrm{fr}}}
$$

with $\dot{\Delta}_{\mathrm{fr}}>0$ the frictional dissipation. Let the internal forces power be decomposed into a reversible part $\dot{U}_{\text {int }}$ and an irreversible part $\dot{\Delta}_{\text {int }} \geqslant 0$ (plastic dissipation, ...) with $\dot{W}_{\text {int }}=$ $\dot{U}_{\text {int }}+\dot{\Delta}_{\text {int }}$. Let $E$ be the system energy with $E \equiv K+U_{\text {int }}$, where $K$ is the kinetic energy. Therefore, assuming pure Neumann boundary conditions (i.e. $\mathbb{S}_{\vec{x}}=\emptyset$ ), if $\delta \vec{x}=\dot{\vec{x}}$ and using Eqs. (12) and (13), Eq. (7) leads to the first thermodynamics principle

$$
\dot{E}=-\dot{\Delta}_{\text {fr }}-\dot{\Delta}_{\text {int }} \quad \forall t \in \mathbb{T} .
$$




\subsection{Finite-elements decomposition}

Thanks to Eq. (4), the terms of Eq. (7) can be rewritten such that

$$
\begin{aligned}
& \delta K=\sum_{i=1,2} \int_{\mathbb{V}_{0}^{i}}\left\{\rho_{0} \varphi^{\xi_{i}} \varphi^{\mu_{i}}\right\} \mathrm{d} \mathbb{V}_{0}^{i}[\ddot{\vec{x}}]^{\mu_{i}} \cdot \delta \vec{x}^{\xi_{i}}=\sum_{i=1,2} M^{\xi_{i} \mu_{i}}[\ddot{\vec{x}}]^{\mu_{i}} \cdot \delta \vec{x}^{\xi_{i}}, \\
& \delta W_{\text {cont }}=\sum_{i=1,2} \int_{\mathbb{S}_{\vec{T}}^{i}}\left\{\vec{T}_{S^{i}} \varphi^{\xi_{i}}\right\} \mathrm{d} \mathbb{S}^{i} \cdot \delta \vec{x}^{\xi_{i}}=\sum_{i=1,2}\left[\vec{F}_{\text {cont }}\right]^{\xi_{i}} \cdot \delta \vec{x}^{\xi_{i}}, \\
& \delta W_{\text {int }}=\sum_{i=1,2} \int_{\mathbb{V}_{0}^{i}}\left\{\Sigma^{\mathrm{T}}\left[\frac{\partial \varphi^{\xi_{i}}}{\partial \vec{x}}\right]^{\mathrm{T}} J\right\} \mathrm{d} \mathbb{V}_{0}^{i} \cdot \delta \vec{x}^{\xi_{i}}=\vec{F}_{\text {int }}^{\xi_{i}} \cdot \delta \vec{x}^{\xi_{i}},
\end{aligned}
$$

where $M^{\xi_{i} \mu_{i}}$ is the mass related to nodes $\xi_{i}$ and $\mu_{i}$. Since $\delta \vec{x} \in \mathbb{F}^{i}$ is an arbitrary vector, Eq. (7) leads to the balance equation

$$
M^{\xi_{i} \mu_{i}}[\ddot{\ddot{x}}]^{\mu_{i}}=\left[\vec{F}_{\text {cont }}-\vec{F}_{\text {int }}\right]^{\xi_{i}} \quad \forall t \in \mathbb{T} \quad \forall i=1,2 .
$$

To be able to integrate this relation in time, $\mathbb{T}$ is decomposed into some intervals $\left[t^{n}, t^{n+1}\right]$ such that $\mathbb{T}=\bigcup_{n=0}^{n=n^{\prime}}\left[t^{n}, t^{n+1}\right]$. Let $\Delta t=t^{n+1}-t^{n}$ be the time step size. Superscripts $n$ and $n+1$ will refer to configurations in time $t^{n}$ and $t^{n+1}$. To be consistent, the time integration must verify Eqs. (9), (11) and (14) (conservation equations).

\subsection{Time integration}

Once the balance Eq. (16) is established for any time $t$, this relation must be integrated in time. To achieve this goal Simo and Tarnow have developed the EMCA method [6]. In this section, to ease notations, we omit the subscript $i$ on the node number $\xi_{i}$. Relations between positions, velocities and accelerations at node $\xi$ becomes

$$
\begin{aligned}
& {\left[\vec{x}^{n+1}\right]^{\xi}=\left[\vec{x}^{n}\right]^{\xi}+\frac{\Delta t}{2}\left[\dot{\vec{x}}^{n+1}\right]^{\xi}+\frac{\Delta t}{2}\left[\dot{\vec{x}}^{n}\right]^{\xi},} \\
& {\left[\dot{\vec{x}}^{n+1}\right]^{\xi}=\left[\dot{\vec{x}}^{n}\right]^{\xi}+\frac{\Delta t}{2}\left[\ddot{\vec{x}}^{n+1}\right]^{\xi}+\frac{\Delta t}{2}\left[\ddot{\vec{x}}^{n}\right]^{\xi} .}
\end{aligned}
$$

These relations are second-order approximations in $\Delta t$. If $\vec{F}_{\text {int }}^{n+1 / 2}$ is a second-order approximation of $\vec{F}_{\text {int }}\left(t^{n+1 / 2}\right)$, the balance Eq. (16) is discretized into a second-order approximation by

$$
\frac{1}{2} M^{\xi \mu}\left[\ddot{\vec{x}}^{n+1}+\ddot{\vec{x}}^{n}\right]^{\mu}=\left[\vec{F}_{\text {cont }}^{n+1 / 2}-\vec{F}_{\text {int }}^{n+1 / 2}\right]^{\xi} .
$$

The internal forces $\left(\vec{F}_{\text {int }}^{n+1 / 2}\right)$ and contact forces $\left(\vec{F}_{\text {cont }}^{n+1 / 2}\right)$ have to be designed to verify the following conservation laws. 


\subsubsection{Conservation of linear momentum}

A sum on $\xi$ in Eq. (18) and the use of Eqs. (17) leads to

$$
\underbrace{\sum_{\xi} M^{\xi \mu}\left[\dot{\vec{x}}^{n+1}\right]^{\mu}}_{\vec{L}^{n+1}}-\underbrace{\sum_{\xi} M^{\xi \mu}\left[\dot{\vec{x}}^{n}\right]^{\mu}}_{\vec{L}^{n}}=\Delta t \sum_{\xi}\left[\vec{F}_{\text {cont }}^{n+1 / 2}-\vec{F}_{\text {int }}^{n+1 / 2}\right]^{\xi},
$$

where the continuous linear momentum $\vec{L}$ defined by Eq. (8) is discretized thanks to Eq. (4) into $\vec{L}=\sum_{\xi} M^{\xi \mu} \dot{\vec{x}}^{\mu}$. Eq. (19) is a discretization of Eq. (9) if

$$
\sum_{\xi}\left[\vec{F}_{\text {int }}^{n+1 / 2}\right]^{\xi}=0 \quad \text { and } \quad \sum_{\xi}\left[\vec{F}_{\text {cont }}^{n+1 / 2}\right]^{\xi}=0 .
$$

\subsubsection{Conservation of angular momentum}

Thanks to Eqs. (17), the vector product between $\vec{x}^{n+1 / 2}=\left(\vec{x}^{n}+\vec{x}^{n+1}\right) / 2$ and Eq. (18) leads to

$$
\frac{1}{\Delta t} \underbrace{M^{\xi \mu}\left[\vec{x}^{n+1}\right]^{\xi} \wedge\left[\dot{\vec{x}}^{n+1}\right]^{\mu}}_{\vec{J}^{n+1}}-\frac{1}{\Delta t} \underbrace{M^{\xi \mu}\left[\vec{x}^{n}\right]^{\xi} \wedge\left[\dot{\vec{x}}^{n}\right]^{\mu}}_{\vec{J}^{n}}=\left[\vec{x}^{n+1 / 2}\right]^{\xi} \wedge\left[\vec{F}_{\text {cont }}^{n+1 / 2}-\vec{F}_{\text {int }}^{n+1 / 2}\right]^{\xi},
$$

where the continuous angular momentum $\vec{J}$ defined by Eq. (10) is discretized thanks to Eq. (4) into $\vec{J}=M^{\xi \mu} \vec{x}^{\xi} \wedge \dot{\vec{x}}^{\mu}$. Therefore, Eq. (21) is a discretization of Eq. (10) if

$$
\left[\frac{\vec{x}^{n+1}+\vec{x}^{n}}{2}\right]^{\xi} \wedge\left[\vec{F}_{\text {int }}^{n+1 / 2}\right]^{\xi}=0 \text { and } \quad\left[\frac{\vec{x}^{n+1}+\vec{x}^{n}}{2}\right]^{\xi} \wedge\left[\vec{F}_{\text {cont }}^{n+1 / 2}\right]^{\xi}=0 .
$$

2.4.3. Conservation of energy

Thanks to Eqs. (17), the dot product between $\dot{\vec{x}}^{n+1 / 2}=\left(\dot{\vec{x}}^{n}+\dot{\vec{x}}^{n+1}\right) / 2$ and Eq. (18) leads to

$$
\underbrace{\frac{M^{\xi \mu}}{2}\left[\dot{\vec{x}}^{n+1}\right]^{\xi} \cdot\left[\dot{\vec{x}}^{n+1}\right]^{\mu}}_{K^{n+1}}-\underbrace{\frac{M^{\xi \mu}}{2}\left[\dot{\vec{x}}^{n}\right]^{\xi} \cdot\left[\dot{\vec{x}}^{n}\right]^{\mu}}_{K^{n}}=\left[\vec{x}^{n+1}-\vec{x}^{n}\right]^{\xi} \cdot\left[\vec{F}_{\text {cont }}^{n+1 / 2}-\vec{F}_{\text {int }}^{n+1 / 2}\right]^{\xi},
$$

where the continuous kinetic energy $K$ defined in Eq. (12) is discretized thanks to Eq. (4) into $K=\frac{1}{2} M^{\xi} \mu \dot{\vec{x}}^{\xi} \cdot \dot{\vec{x}}^{\mu}$. Let $E$ be the discretized energy, let $U_{\text {int }}$ be the discretized internal energy, let $W_{\text {int }}$ be the discretized work of the internal forces, let $\Delta_{\text {int }} \geqslant 0$ be the discretized internal dissipation during the step and let $\Delta_{\mathrm{fr}} \geqslant 0$ be the discretized friction dissipation during the step. Therefore Eq. (14) is discretized into

$$
E^{n+1}-E^{n}=-\Delta_{\text {fr }}-\Delta_{\text {int }} .
$$

If this latest expression is compared with Eq. (23), the internal forces must lead to

$$
\left[\vec{F}_{\text {int }}^{n+1 / 2}\right]^{\xi} \cdot\left[\vec{x}^{n+1}-\vec{x}^{n}\right]^{\xi}=U_{\text {int }}^{n+1}-U_{\text {int }}^{n}+\Delta_{\text {int }}
$$

and the contact forces must lead to

$$
\left[\vec{F}_{\text {cont }}^{n+1 / 2}\right]^{\xi} \cdot\left[\vec{x}^{n+1}-\vec{x}^{n}\right]^{\xi}=W_{\text {cont }}^{n+1}-W_{\text {cont }}^{n}-\Delta_{\mathrm{fr}} .
$$


In this latest expression, we have introduced $W_{\text {cont }}$, which is tolerated to be lower than zero during the persistent contact but which must be equal to zero when the contact is released. This methodology was proposed by Armero and Petöcz [7].

The problem of the EMCA algorithm is to find a consistent expression of the internal/contact forces that verifies the conservation conditions. A formulation of the internal forces for hyperelastic models was given by Gonzalez [19] and extended to dynamic finite deformation plasticity, with a hyperelastic formulation, by Meng and Laursen [20]. In a previous work [21,22], we have developed a new expression of the internal forces valid for an elasto-plastic hypoelastic model, which verifies these conditions. The goal of this paper is to establish the expression of the contact forces that verify Eqs. (20), (22) and (26).

\subsubsection{Numerical dissipation}

When the number of degrees of freedom increases, the numerical modes of high frequency can lead to divergence of the time integration. Therefore, some controlled numerical dissipation can be beneficial. This was first introduced in this EMCA algorithm by Armero and Romero [23,24], leading to the energy dissipative momentum conserving algorithm (EDMC). In this EDMC scheme, Eqs. (17) and (18) are rewritten as

$$
\begin{aligned}
& {\left[\vec{x}^{n+1}\right]^{\xi}=\left[\vec{x}^{n}\right]^{\xi}+\frac{\Delta t}{2}\left[\dot{\vec{x}}^{n+1}\right]^{\xi}+\frac{\Delta t}{2}\left[\dot{\vec{x}}^{n}\right]^{\xi}+\Delta t\left[\vec{G}_{\text {diss }}^{n+1 / 2}\right]^{\xi},} \\
& {\left[\dot{\vec{x}}^{n+1}\right]^{\xi}=\left[\dot{\vec{x}}^{n}\right]^{\xi}+\frac{\Delta t}{2}\left[\ddot{\vec{x}}^{n+1}\right]^{\xi}+\frac{\Delta t}{2}\left[\ddot{\vec{x}}^{n}\right]^{\xi},} \\
& \frac{1}{2} M^{\xi \mu}\left[\ddot{\vec{x}}^{n+1}+\ddot{\vec{x}}^{n}\right]^{\mu}=\left[\vec{F}_{\text {cont }}^{n+1 / 2}-\vec{F}_{\text {int }}^{n+1 / 2}-\vec{F}_{\text {diss }}^{n+1 / 2}\right]^{\xi}
\end{aligned}
$$

with $\vec{G}_{\text {diss }}^{n+1 / 2}$ and $\vec{F}_{\text {diss }}^{n+1 / 2}$ respectively the dissipation velocities and the dissipation forces. Proceeding as in Sections 2.4.1-2.4.3, the dissipation forces have to verify

$$
\sum_{\xi}\left[\vec{F}_{\text {diss }}^{n+1 / 2}\right]^{\xi}=0 \quad \text { and } \quad\left[\frac{\vec{x}^{n+1}+\vec{x}^{n}}{2}\right]^{\xi} \wedge\left[\vec{F}_{\text {diss }}^{n+1 / 2}\right]^{\xi}=0
$$

to verify the conservation of the linear and angular momentum, and have to verify

$$
\left[\vec{F}_{\text {diss }}^{n+1 / 2}\right]^{\xi} \cdot\left[\vec{x}^{n+1}-\vec{x}^{n}\right]^{\xi}=\Delta_{F} \geqslant 0
$$

to dissipate numerically the energy $\Delta_{F}$ (part of the energy dissipated by the dissipation forces). The dissipation velocities have to verify

$$
M^{\xi} \mu\left[\vec{G}_{\text {diss }}^{n+1 / 2}\right]^{\xi} \wedge\left[\frac{\dot{\vec{x}}^{n+1}+\dot{\vec{x}}^{n}}{2}\right]^{\mu}=0
$$

to verify conservation of the angular momentum (the linear momentum does not depend on the dissipation velocities), and have to verify

$$
M^{\xi \mu}\left[\dot{\vec{x}}^{n+1}-\dot{\vec{x}}^{n}\right]^{\mu} \cdot\left[\vec{G}_{\text {diss }}^{n+1 / 2}\right]^{\xi}=\Delta_{G} \geqslant 0
$$

to dissipate numerically the energy $\Delta_{G}$ (part of the energy dissipated by the dissipation velocities). Let us note that both vectors have to be simultaneously considered to avoid bifurcation in the 
spectral matrix analysis $[23,24]$. Depending on the form of these vectors, the EDMC scheme can be first-order (EDMC-1) or second-order (EDMC-2) accurate. For first-order accurate schemes, the dissipation velocities can be expressed at node $\xi$ as [23] (no sum on $\xi$ )

$$
\left[\vec{G}_{\text {diss }}^{n+1 / 2}\right]^{\xi}=\chi \frac{\left\|\left[\dot{\vec{x}}^{n+1}\right]^{\xi}\right\|-\left\|\left[\dot{\vec{x}}^{n}\right]^{\xi}\right\|}{\left\|\left[\dot{\vec{x}}^{n+1}\right]^{\xi}\right\|+\left\|\left[\dot{\vec{x}}^{n}\right]^{\xi}\right\|} \frac{\left[\dot{\vec{x}}^{n+1}+\dot{\vec{x}}^{n}\right]^{\xi}}{2},
$$

where $\chi$ is a parameter that controls the amount of numerical dissipation. Let us assume that in the linear range (small displacement) the internal forces are evaluated thanks to a stiffness secondorder tensor $\mathbf{K}$. Then the internal forces are reduced to

$$
\left[\vec{F}_{\text {int }}^{n+1 / 2}\right]^{\xi}=\mathbf{K}^{\xi \mu} \frac{\left[\vec{x}^{n+1}+\vec{x}^{n}\right]^{\mu}}{2} .
$$

Therefore, to avoid a bifurcation in the spectral radius analysis $[23,24]$, the dissipation forces have to reduce themselves, in the linear range, to

$$
\left[\vec{F}_{\text {diss }}^{n+1 / 2}\right]^{\xi}=\chi \mathbf{K}^{\xi \mu} \frac{\left[\vec{x}^{n+1}-\vec{x}^{n}\right]^{\mu}}{2} .
$$

In [25], we have proposed an expression of the dissipation forces for hypoelastic models subjected to plastic deformations. A hypoelastic formulation computes the stress at the actual configuration from a stress increment. This stress increment takes into account both the elastic part and the plastic part of the deformations. For more details on this model we refer to Ponthot [26]. In this paper we will present an expression of the dissipation forces related to the contact forces.

\section{Formulation of the node-on-segment contact}

Let us consider that the nodes on the surface $\mathbb{S}^{1}$ are the slave nodes and that surface $\mathbb{S}^{2}$ is the master surface. By inverting this convention at each step, one gets a double pass algorithm. Surface $\mathbb{S}^{2}$ can be represented by a two-component vector $u: \mathbb{S}_{0}^{2} \times \mathbb{T} \rightarrow \mathbb{R}^{2}$ (the components are $u_{1}$ and $u_{2}$ ). Therefore, ones gets $\vec{y}(u, t): \mathbb{S}_{0}^{2} \times \mathbb{T} \rightarrow \mathbb{X}^{2}$ with $\mathbb{X}^{2}$ defined by Eq. (2). Let us consider a point $\vec{x}_{0} \in \mathbb{V}_{0}^{1}$ associated with a node $\vec{x}^{\xi_{1}}=\vec{x}\left(\vec{x}_{0}, t\right)$. Let us define $\vec{y}\left(\vec{x}_{0}, t\right)$ the closest point projection of $\vec{x}^{\xi_{1}}$ on the master surface (Fig. 1). Using the discretization of the master surface $\vec{y}\left(\vec{x}_{0}, t\right)=\varphi^{\xi_{2}}\left(u\left(\vec{x}_{0}, t\right)\right) \vec{x}^{\xi_{2}}$, it yields

$$
\begin{aligned}
& \frac{\partial \vec{x}\left(\vec{x}_{0}, t\right)}{\partial t}=\dot{\vec{x}}^{\xi_{1}}, \\
& \frac{\partial \vec{y}\left(\vec{x}_{0}, t\right)}{\partial t}=\vec{t}_{\alpha}\left(u\left(\vec{x}_{0}, t\right)\right) \frac{\partial u_{\alpha}\left(\vec{x}_{0}, t\right)}{\partial t}+\varphi^{\xi_{2}}\left(u\left(\vec{x}_{0}, t\right)\right) \dot{\vec{x}}^{\xi_{2}},
\end{aligned}
$$

where $\vec{t}_{\alpha}\left(u\left(\vec{x}_{0}, t\right)\right)=\partial \vec{y}\left(u\left(\vec{x}_{0}, t\right)\right) / \partial u_{\alpha}=\left(\partial \varphi^{\xi_{2}} / \partial u_{\alpha}\right) \vec{x}^{\xi_{2}}$ is the tangent vector of the surface.

\subsection{Derivation of the gap}

In this section we derive the normal gap and the tangential gap with respect to $\vec{x}_{0}$ constant (material derivation) [27]. These derivations will be necessary to establish the contact forces 
formulation. Let us define $g\left(\vec{x}_{0}, t\right): \mathbb{S}_{0}^{1} \times \mathbb{T} \rightarrow \mathbb{R}$ the normal gap such that

$$
g\left(\vec{x}_{0}, t\right)=\left[\vec{x}\left(\vec{x}_{0}, t\right)-\vec{y}\left(\vec{x}_{0}, t\right)\right] \cdot \vec{n}\left(\vec{x}_{0}, t\right) .
$$

The normal has a unit norm leading to $\vec{n}\left(\vec{x}_{0}, t\right) \cdot \partial \vec{n}\left(\vec{x}_{0}, t\right) / \partial t=0$. Since the normal is perpendicular to the tangent plane, it leads to $\vec{t}_{\alpha}\left(u\left(\vec{x}_{0}, t\right)\right) \frac{\partial u_{\alpha}}{\partial t} \cdot \vec{n}\left(\vec{x}_{0}, t\right)=0$. Let us note that the normal gap is not equal to zero during the contact since we tolerate a penetration. Therefore Eqs. (35) and (36) lead to

$$
\frac{\partial g\left(\vec{x}_{0}, t\right)}{\partial t}=\left[\dot{\vec{x}}^{\xi_{1}}-\varphi^{\xi_{2}}\left(u\left(\vec{x}_{0}, t\right)\right) \dot{\vec{x}}^{\xi_{2}}\right] \cdot \vec{n}\left(\vec{x}_{0}, t\right) .
$$

Now let us derive the projection of the gap on the tangents. Since the projection is orthogonal, one gets

$$
\left[\vec{x}\left(\vec{x}_{0}, t\right)-\vec{y}\left(\vec{x}_{0}, t\right)\right] \cdot t_{\alpha}\left(\vec{x}_{0}, t\right)=0 \quad \text { with } \alpha=1,2 .
$$

This expression is derived, using Eq. (35), into [27]

$$
\underbrace{\left[\dot{\vec{x}}^{\xi_{1}}-\varphi^{\xi_{2}} \dot{\vec{x}}^{\xi_{2}}\right] \cdot \vec{t}_{\alpha}+g \vec{n} \cdot \vec{t}_{\alpha, t}}_{\equiv B_{\alpha}}=\underbrace{\left[\vec{t}_{\beta} \cdot \vec{t}_{\alpha}-g \vec{n} \cdot \vec{t}_{\alpha, \beta}\right]}_{\equiv A_{\alpha \beta}} \dot{u}_{\beta}
$$

with $\vec{t}_{\alpha, t}=\left(\partial \varphi^{\xi_{2}} / \partial u_{\alpha}\right) \dot{\vec{x}}^{\xi_{2}}$ and $\vec{t}_{\alpha, \beta}=\left(\partial^{2} \varphi^{\xi_{2}} / \partial u_{\beta} \partial u_{\alpha}\right) \vec{x}^{\xi_{2}}$.

\subsection{Expression of the contact forces}

The pressure at node $\xi_{1}$ is expressed by $t_{N}\left(\vec{x}_{0}^{\xi^{1}}, t\right): \mathbb{S}_{0}^{1} \times \mathbb{T} \rightarrow \mathbb{R}^{+}$. The Kuhn-Tucker conditions are expressed by

$$
g\left(\vec{x}_{0}, t\right) \geqslant 0 \quad \text { and } \quad t_{N}\left(\vec{x}_{0}, t\right) \geqslant 0 \quad \text { and } \quad t_{N}\left(\vec{x}_{0}, t\right) g(\vec{x}, t)=0 .
$$

The tangential pressure is computed in a dual base $\vec{t}_{d_{1}}, \vec{t}_{d_{2}}$ of $\vec{t}_{1}, \vec{t}_{2}$ [27] defined by

$$
\vec{t}_{d_{\alpha}} \equiv\left[\vec{t}_{\alpha} \cdot \vec{t}_{\beta}\right]^{-1} \vec{t}_{\beta} \quad \text { with } \vec{t}_{d_{\alpha}} \cdot \vec{t}_{\beta}=\delta_{\alpha \beta}
$$

where $\left[\vec{t}_{\alpha} \cdot \vec{t}_{\beta}\right]^{-1}$ represents the component $\alpha \beta$ of the inverse of the 2-2 matrix $\vec{t}_{\alpha} \cdot \vec{t}_{\beta}$. Therefore, let $\vec{t}_{T_{1}}\left(\vec{x}_{0}, t\right): \mathbb{S}_{0}^{1} \times \mathbb{T} \rightarrow \mathbb{R}$ be the friction pressure along $\vec{t}_{d_{1}}$ and let $\vec{t}_{T_{2}}\left(\vec{x}_{0}, t\right): \mathbb{S}_{0}^{1} \times \mathbb{T} \rightarrow \mathbb{R}$ be the friction pressure along $\vec{t}_{d_{2}}$. The friction pressure $\vec{T}$ is therefore computed from

$$
\vec{T} \equiv t_{T_{\alpha}} \vec{t}_{d_{\alpha}} \quad \text { with }\|\vec{T}\|=\sqrt{t_{T_{\alpha}} t_{T_{\beta}}\left[\vec{t}_{\alpha} \cdot \vec{t}_{\beta}\right]^{-1}} .
$$

Let us define $\vec{v}_{T}$ the slip velocity, $\gamma_{c}$ the slip rate, and $\Phi_{c} \equiv\|\vec{T}\|-\mu_{c} t_{N}$ the Coulomb criterion ( $\mu_{c}$ is the friction coefficient). Therefore the friction conditions are

$$
\vec{v}_{T}=\dot{u}_{\beta} \vec{t}_{\beta}=-\gamma_{c} \frac{\vec{T}}{\|\vec{T}\|} \quad \text { and } \quad \Phi_{c} \leqslant 0 \quad \text { and } \quad \gamma_{c} \geqslant 0 \quad \text { and } \quad \gamma_{c} \Phi_{c}=0 .
$$

Using Eqs. (3) and (35), $\delta W_{\text {cont }}$ defined in Eq. (7) can be rewritten as

$$
\delta W_{\text {cont }}=\int_{\mathbb{S}^{1}(t)}\left\{\left[t_{N} \vec{n}+\vec{T}\right] \cdot\left[\delta \vec{x}^{\xi_{1}}-\varphi_{\delta}\left(\vec{x}_{0}, t\right) \delta \vec{x}^{\xi_{2}}\right]\right\} \mathrm{d} \mathbb{S}^{1}(t) .
$$


Using Eqs. (37) and (39), with $g=0$ (that corresponds to verify the Kuhn-Tucker relations defined by Eq. (40)), Eq. (44) leads to

$$
\delta W_{\text {cont }}=\int_{\mathbb{S}^{1}(t)}\left\{t_{N} \delta g+t_{T_{\alpha}} \delta u_{\alpha}\right\} \mathrm{d}^{1}(t) .
$$

Now using Eqs. (37) and (39), with $g \neq 0$ (that corresponds to the actual situation), allows us to rewrite Eq. (45) into

$$
\begin{aligned}
\delta W_{\text {cont }}= & \int_{\mathbb{S}^{1}(t)}\left\{t_{N} \vec{n} \cdot\left[\delta \vec{x}^{\xi_{1}}-\varphi^{\xi_{2}}\left(\vec{x}^{\xi_{1}}\right) \delta \vec{x}^{\xi_{2}}\right]\right\} \mathrm{d} \mathbb{S}^{1}(t) \\
& +\int_{\mathbb{S}^{1}(t)}\left\{t_{T_{\alpha}} A_{\beta \alpha}^{-1}\left[\delta \vec{x}^{\xi_{1}}-\varphi^{\xi_{2}}\left(\vec{x}^{\xi_{1}}\right) \delta \vec{x}^{\xi_{2}}\right] \cdot \vec{t}_{\beta}\right\} \mathrm{d} \mathbb{S}^{1}(t) \\
& +\int_{\mathbb{S}^{1}(t)}\left\{t_{T_{\alpha}} A_{\beta \alpha}^{-1} g \vec{n} \cdot \frac{\partial \varphi^{\xi_{2}}}{\partial u_{\beta}}\left(\vec{x}^{\xi_{1}}\right) \delta \vec{x}^{\xi_{2}}\right\} \mathrm{d} \mathbb{S}^{1}(t) .
\end{aligned}
$$

Comparing this expression with Eq. (15) leads to the contact forces

$$
\begin{aligned}
& \vec{F}_{\text {cont }}^{\xi_{1}}=t_{N} \vec{n}\left(x^{\xi_{1}}\right)+t_{T_{\alpha}} A_{\beta \alpha}^{-1} \vec{t}_{\beta}\left(x^{\xi_{1}}\right), \\
& \vec{F}_{\text {cont }}^{\xi_{2}}=-t_{N} \varphi^{\xi_{2}}\left(x^{\xi_{1}}\right) \vec{n}\left(x^{\xi_{1}}\right)-t_{T_{\alpha}} A_{\beta \alpha}^{-1} \varphi^{\xi_{2}}\left(x^{\xi_{1}}\right) \vec{t}_{\beta}\left(x^{\xi_{1}}\right)-t_{T_{\alpha}} A_{\beta \alpha}^{-1}\left(\vec{x}^{\xi_{1}}\right) g\left(\vec{x}^{\xi_{1}}\right) \vec{n}\left(\vec{x}^{\xi_{1}}\right) \frac{\partial \varphi^{\xi_{2}}}{\partial u_{\beta}}\left(\vec{x}^{\xi_{1}}\right) .
\end{aligned}
$$

In this expression, we have integrated the surface in the pressure (i.e. $t_{N} \quad \int_{\mathbb{S}^{1}}\left\{t_{N}\right\} \mathrm{d} \mathbb{S}^{1}$ and $\left.t_{T_{\alpha}} \quad \int_{\mathbb{S}^{1}}\left\{t_{T_{\alpha}}\right\} d \mathbb{S}^{1}\right)$. Therefore, $t_{N}$ and $t_{T_{\alpha}}$ correspond to the forces and not to the pressure. This expression of the contact forces will lead to the conservation of the angular momentum (thanks to the term $g \vec{n}$ ) and has been established by Armero and Petöcz [8]. Finally, using Eq. (43), the frictional dissipation defined in Eq. (13) can be deduced from Eq. (45), leading to

$$
\dot{\Delta}_{\mathrm{fr}}=-t_{T_{\alpha}} \dot{u}_{\alpha}=-t_{T_{\alpha}} \delta_{\alpha \beta} \dot{u}_{\beta}=-\vec{T} \cdot \vec{v}_{T}=\mu_{c} t_{N} \gamma_{c}>0 .
$$

Now we will adapt the contact forces (47) to a surface with normal discontinuity.

\subsection{Discontinuous normal algorithm}

When studying the contact between bodies discretized into finite elements, the boundary of each body is composed of bilinear Coons patches (3D elements with linear shape functions) or of linear segments (2D elements with linear shape functions). Therefore, the normal is no longer continuous at the interface between two patches or segments. In this section, we will extend the method proposed by Graillet [5] to solve this problem. This method will lead to a continuous normal $\vec{n}^{c}\left(\vec{x}^{\xi_{1}}, t\right)$ for each slave node $\xi_{1}$, and will determine a master entity (segment or patch) number $n o$ as well as a reduced master continuous coordinates $u^{c}$ in order to uniquely determine a projection for each slave node on the master surface. These two master values have to be considered to deduce the projection point $\vec{y}\left(n o, u^{c}, t\right)$. We will analyze three possible situations: the slave node projects itself out of all the entities (Fig. 2a), the slave node projects itself on one entity (Fig. 2b) or the slave node projects itself on more than one entity (Fig. 2c). 


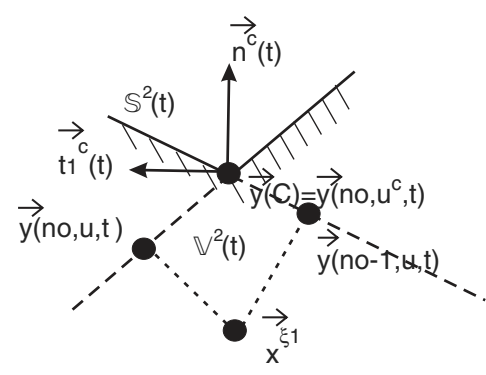

(a)

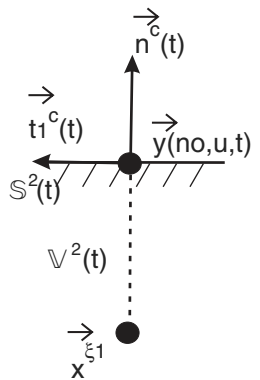

(b)



(c)

Fig. 2. Projection configurations in 2 dimensions: (a) projections are out of the entities; (b) there is one projection; (c) there are two projections.

\subsubsection{Case 1: projections are out of the entities}

Let us suppose a 2D problem with a projection of slave node $\vec{x}^{\xi_{1}}$ on the two segments no-1 and $n o$, but with these two projections out of the effective part of the segments (Fig. 2a). Let $\vec{y}(C)$ be the intersection of these two segments. Normal is continuous with a slave node displacement for

$$
\vec{y}\left(n o, u^{c}, t\right)=\vec{y}(C, t) \quad \text { and } \quad \vec{n}^{c}\left(\vec{x}^{\xi_{1}}, t\right)=\frac{\vec{y}\left(n o, u^{c}, t\right)-\vec{x}^{\xi_{1}}}{\left\|\vec{y}\left(n o, u^{c}, t\right)-\vec{x}^{\xi_{1}}\right\|} .
$$

For the 3D problem, Eqs. (49) are taken in the plane that includes the slave node and that is perpendicular to the intersection edge of the two patches.

\subsubsection{Case 2: there is one projection}

If there is only one projection on entity no for coordinates $u$ (Fig. 2b), we directly have

$$
\vec{y}\left(n o, u^{c}, t\right)=\vec{y}(n o, u, t) \quad \text { and } \quad \vec{n}^{c}\left(\vec{x}^{\xi_{1}}, t\right)=\frac{\vec{y}\left(n o, u^{c}, t\right)-\vec{x}^{\xi_{1}}}{\left\|\vec{y}\left(n o, u^{c}, t\right)-\vec{x}^{\xi_{1}}\right\|} .
$$

\subsubsection{Case 3: there are two or more projections}

If there are more than two projections, let us consider the two closest projections. Let us suppose a $2 \mathrm{D}$ problem with a projection of slave node $\vec{x}^{\xi_{1}}$ on the two segments no-1 and no (Fig. 2c). Let $\alpha_{c}$ be the angle defined in Fig. 2c. If this angle is larger than or equal to $90^{\circ}$ (limit configuration illustrated in Fig. 3a), the slave node has always two projections. But, if we want the normal to evolve continuously, the slave node has to evolve from a simple projection configuration to a double projection configuration. Then, if the node is closer from segment $n o-1$ or no than from the bisecting lines (gray surface on Fig. 3a), we consider that the contact occurs with this segment and the formulation is identical to Eq. (50) (assuming the segment number is no). If the slave node is closer from the bisecting line (hashed zone on Fig. 3a), the normal must be transformed to be continuous. The method proposed by Graillet [5] is the following. Let us consider Fig. 3b, where the angle $\alpha_{c}$ (assumed greater than $60^{\circ}$ as we will explain later) is represented and the complementary angle is decomposed into 4 angles of equal amplitude 


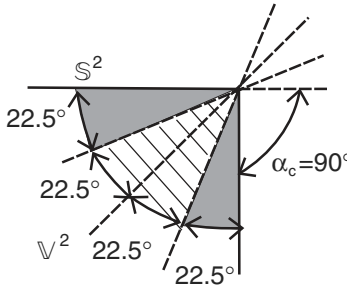

(a)

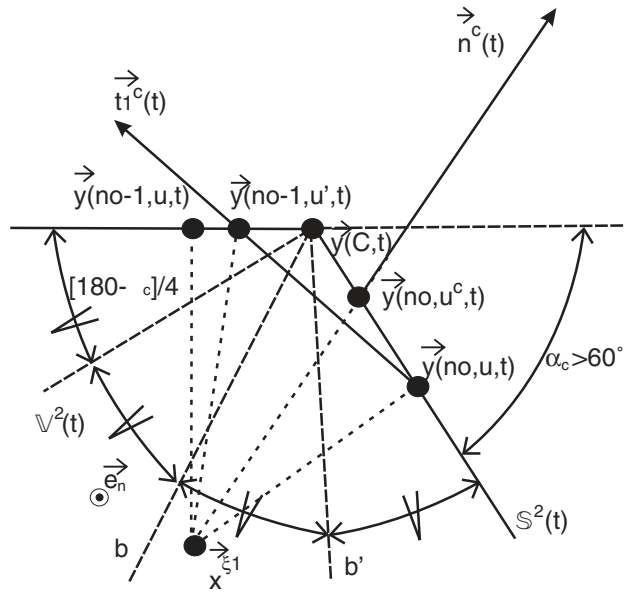

(b)

Fig. 3. Description of the two-projection configuration (2 dimensions): (a) limit configuration $\alpha_{c}=90^{\circ}$; (b) configuration for an angle larger than $60^{\circ}$.

$\left(180^{\circ}-\alpha_{c}\right) / 4$. Let us assume that slave node $\vec{x}^{\xi_{1}}$ is closer from segment no than from segment $n o-1$ (i.e. it is between lines $b$ and $\left.b^{\prime}\right)$. The two projections are $\vec{y}(n o-1, u, t)$ and $\vec{y}(n o, u, t)$. Therefore, projection $\vec{y}(n o-1, u, t)$ is moved to $\vec{y}\left(n o-1, u^{\prime}, t\right)$ such that the normal becomes continuous with

$$
\begin{aligned}
& \vec{n}^{c}\left(\vec{x}^{\xi_{1}}, t\right)=\frac{\vec{y}\left(\text { no }-1, u^{\prime}, t\right)-\vec{y}(n o, u, t)}{\left\|\vec{y}(n o, u, t)-\vec{y}\left(n o-1, u^{\prime}, t\right)\right\|} \wedge \vec{e}_{n}, \\
& \vec{y}\left(n o, u^{c}, t\right)=\left[\vec{x}+a \vec{n}^{c}\left(\vec{x}^{\xi_{1}}, t\right)\right] \cap \mathbb{S}^{2}(t)
\end{aligned}
$$

with $\vec{e}_{n}$ the unit vector perpendicular to the plane and $a$ a scalar that is numerically computed (in a similar way a projection is computed) to reach the intersection. Now we have to determine $\vec{y}\left(n o-1, u^{\prime}, t\right)$ such that $\vec{n}^{c}$ will have a continuous evolution. Let $\vec{y}(C, t)$ be the intersection of the two segments as defined on Fig. $3 b$. Therefore, the system of equations to be solved is

$$
\begin{aligned}
& \vec{y}\left(\text { no }-1, u^{\prime}, t\right)=\vec{y}(C, t)+\alpha^{\prime} L^{\prime} \frac{\vec{y}(\text { no }-1, u, t)-\vec{y}(C, t)}{\|\vec{y}(n o-1, u, t)-\vec{y}(C, t)\|}, \\
& L^{\prime}=\|\vec{y}(n o, u, t)-\vec{y}(C, t)\|,
\end{aligned}
$$

where $\alpha^{\prime}$ is determined to lead to a normal continuous evolution. To achieve that goal, if the slave node evolves from line $b$ to line $b^{\prime}$ (Fig. $3 \mathrm{~b}$ ), the normal has to evolve from a direction parallel to $b$ to a direction perpendicular to segment no. Then, point $\vec{y}\left(n o-1, u^{\prime}, t\right)$ has to evolve from $\vec{y}(n o-$ $1, u, t)$ to $\vec{y}(C, t)$ and, actually, $\alpha^{\prime}$ has to evolve from unity to zero, yielding

$$
\begin{aligned}
& \alpha^{\prime}=\frac{\|\vec{y}(n o, u, t)-\vec{x}\| / L^{\prime}-\tan \left(\left(\pi-\alpha_{c}\right) / 4\right)}{\tan \left(\left(\pi-\alpha_{c}\right) / 2\right)-\tan \left(\left(\pi-\alpha_{c}\right) / 4\right)} \text { with } \tan \left(\frac{\pi-\alpha_{c}}{2}\right)=\sqrt{\frac{1+\cos \alpha_{c}}{1-\cos \alpha_{c}}} \text { and } \\
& \tan \left(\frac{\pi-\alpha_{c}}{4}\right)=\sqrt{\frac{1-\sqrt{\left(1+\cos \alpha_{c}\right) / 2}}{1+\sqrt{\left(1+\cos \alpha_{c}\right) / 2}}} .
\end{aligned}
$$




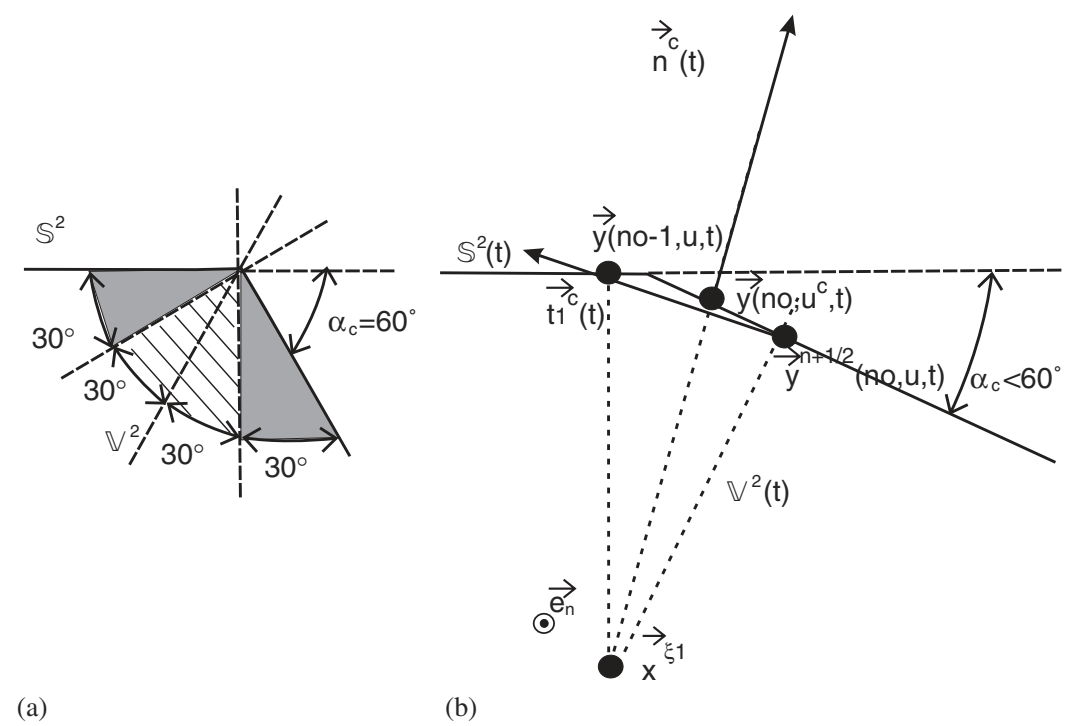

Fig. 4. Description of the two-projection configuration (2 dimensions): (a) limit configuration $\alpha_{c}=60^{\circ}$; (b) configuration for an angle larger than $60^{\circ}$.

Although the above formula is correct for all angles $\alpha_{c}$, we have assumed that $\alpha_{c}$ is larger than $60^{\circ}$. In fact, for such a limit configuration represented at Fig. $4 \mathrm{a}$, if there are two projections, the slave node is always closer from bisecting line $b$ than from the segments. Therefore, the methodology that gives a continuous normal could be simplified by taking a normal to the line that joins the two projections as represented in Fig. 4b. The intersection between the surface and the line directed along the normal and originating from the slave node gives the actual projection point. With $\vec{e}_{n}$ the vector perpendicular to the plane and with $a$ a scalar numerically computed (in a similar way a projection is computed), assuming that the intersection occurs with segment no, one gets

$$
\begin{aligned}
& \vec{n}^{c}\left(\vec{x}^{\xi_{1}}, t\right)=\frac{\vec{y}(\text { no }-1, u, t)-\vec{y}(n o, u, t)}{\|\vec{y}(n o, u, t)-\vec{y}(n o-1, u, t)\|} \wedge \vec{e}_{n}, \\
& \vec{y}\left(n o, u^{c}, t\right)=\left[\vec{x}+a \vec{n}^{c}\left(\vec{x}^{\xi_{1}}, t\right)\right] \cap \mathbb{S}^{2}(t) .
\end{aligned}
$$

When studying a 3D problem, all the formulas developed remain correct when applied in the plane including $\vec{x}^{\xi_{1}}, \vec{y}(n o-1, u, t)$ and $\vec{y}(n o, u, t)$. Moreover, in Figs. 2a, $3 \mathrm{~b}$ and $4 \mathrm{~b}$, we have represented the continuous tangent $t_{1}^{c}$ associated with the continuous normal $n^{c}$.

\section{Consistent contact forces formulation}

In this section we propose an original implementation of the method proposed by Armero and Petöcz $[7,8]$ that leads to verify the conservation laws. Our implementation allows us to take into account the discontinuity of the normal. Let us work in configuration $n+\frac{1}{2}$, which is obtained 
with the positions $\vec{x}^{n+1 / 2}=\left(\vec{x}^{n}+\vec{x}^{n+1}\right) / 2$. The algorithm developed in Section 3.3, and applied in this configuration, leads to the continuous normal $\vec{n}^{c}\left(\vec{x}^{\xi_{1}}, t^{n+1 / 2}\right)$ for each slave node $\xi_{1}$ and to a unique projection on a segment number no and with coordinates $u^{c n+1 / 2}$. Moreover, since the 3-dimensional boundary is composed of Coons patches, to be able to consider frictional forces when the slave node moves from one patch to another, the contact formulation is modified. We rewrite the formulations that depend on the curvilinear coordinates, such that the formulations depend only on the nodes projections. For a complex surface, it is always easier to compute expressions that depend on the closest projection than to compute expressions that depend on curvilinear coordinates.

\subsection{Normal forces}

Eq. (37) is integrated in time by the use of a finite difference stencil, leading to the dynamic gap defined by Armero and Petöcz [7]:

$$
g_{d}^{n+1}=g_{d}^{n}+\vec{n}^{c}\left(\vec{x}^{\xi_{1}}, t^{n+1 / 2}\right) \cdot\left[\vec{x}^{n+1}-\vec{x}^{n}\right]^{\xi_{1}}-\vec{n}^{c}\left(\vec{x}^{\xi_{1}}, t^{n+1 / 2}\right) \cdot\left[\vec{y}^{n+1}\left(n o, u^{c n+1 / 2}\right)-\vec{y}^{n}\left(n o, u^{c n+1 / 2}\right)\right],
$$

where we use the coordinate $u^{c n+1 / 2}$ obtained in the mid-configuration to evaluate the projection in configurations $n$ and $n+1$. The dynamic gap is initialized with the true gap (i.e. $g^{n}=\left[\vec{x}^{n}-\vec{y}^{n}\right] \cdot \vec{n}^{n}>0$ ) before the first contact detection (i.e. $g^{n+1}=\left[\vec{x}^{n+1}-\vec{y}^{n+1}\right] \cdot \vec{n}^{n+1} \leqslant 0$ ) (see [7] for details). Using a normal penalty $k_{N}$, a potential of contact was defined by Armero and Petöcz [7]:

$$
U(g)=\frac{1}{2} k_{N} g^{2} \quad \text { if } g \leqslant 0 \quad \text { and } \quad U(g)=0 \quad \text { if } g>0,
$$

leading to the evaluation of the normal force

$$
\begin{aligned}
& t_{N}\left(\vec{x}^{\xi_{1}}, t^{n+1 / 2}\right)=-\frac{U\left(g_{d}^{n+1}\right)-U\left(g_{d}^{n}\right)}{g_{d}^{n+1}-g_{d}^{n}} \quad \text { if } g_{d}^{n+1} \neq g_{d}^{n} \\
& =-\frac{\partial U}{\partial g}\left(\frac{g_{d}^{n+1}+g_{d}^{n}}{2}\right) \quad \text { if } g_{d}^{n+1}=g_{d}^{n} .
\end{aligned}
$$

The normal components of the contact forces defined in Eq. (47) are then obtained by

$$
\begin{aligned}
& {\left[\vec{F}_{\text {cont }}^{n+1 / 2}\right]_{N}^{\xi_{1}}=t_{N}\left(\vec{x}^{\xi_{1}}, t^{n+1 / 2}\right) \vec{n}^{c}\left(\vec{x}^{\xi_{1}}, t^{n+1 / 2}\right),} \\
& {\left[\vec{F}_{\text {cont }}^{n+1 / 2}\right]_{N}^{\xi_{2}}=-t_{N}\left(x^{\xi_{1}}, t^{n+1 / 2}\right) \varphi_{\delta}^{\xi_{2}}\left(n o, u^{c n+1 / 2}\right) \vec{n}^{c}\left(\vec{x}^{\xi_{1}}, t^{n+1 / 2}\right) .}
\end{aligned}
$$

\subsubsection{Conservation of linear momentum}

Since $\left[1-\sum_{\xi_{2}} \varphi^{\xi_{2}}\left(n o, u^{c n+1 / 2}\right)\right]=0$, Eq. (20) is directly verified from Eq. (58) that leads to

$$
\left[\vec{F}_{\text {cont }}^{n+1 / 2}\right]_{N}^{\xi_{1}}+\sum_{\xi_{2}}\left[\vec{F}_{\text {cont }}^{n+1 / 2}\right]_{N}^{\xi_{2}}=0
$$




\subsubsection{Conservation of angular momentum}

Expressions (58) verify Eq. (22) since

$$
\begin{aligned}
& \sum_{i=1,2}\left[\frac{\vec{x}^{n+1}+\vec{x}^{n}}{2}\right]_{N}^{\xi_{i}} \wedge\left[\vec{F}_{\text {cont }}^{n+1 / 2}\right]_{N}^{\xi_{i}} \\
& =-t_{N}\left(\vec{x}^{\xi_{1}}, t^{n+1 / 2}\right) \vec{n}^{c}\left(\vec{x}^{\xi_{1}}, t^{n+1 / 2}\right) \wedge \underbrace{\left[\left[\frac{\vec{x}^{n+1}+\vec{x}^{n}}{2}\right]^{\xi_{1}}-\varphi_{\delta}^{\xi_{2}}\left(n o, u^{c n+1 / 2}\right)\left[\frac{\vec{x}^{n+1}+\vec{x}^{n}}{2}\right] \xi_{2}\right]}_{=g\left(\vec{x}^{\xi_{1}}, t^{n+1 / 2}\right) \vec{n}^{c}\left(\vec{x}^{\xi_{1}}, t^{n+1 / 2}\right)}=0 .
\end{aligned}
$$

\subsubsection{Conservation of energy}

Let us study the cycle defined by Armero and Petöcz [7]: in configuration 1 there is no contact $\left(g_{d}^{1}=g^{1}>0\right)$, in configuration $n$ with $2 \leqslant n \leqslant n^{\prime}$ there is a persistent contact $\left(g_{d}^{n} \leqslant 0\right)$, and in configuration $n^{\prime}+1$ the contact is released $\left(g_{d}^{n^{\prime}+1}>0\right)$. Let us first note that, using Eqs. (55) and (58), for each $1 \leqslant n \leqslant n^{\prime}$ we have

$$
\begin{aligned}
W_{\mathrm{cont}}^{n+1}-W_{\mathrm{cont}}^{n} & =\left[\vec{F}_{\mathrm{cont}}^{n+1 / 2}\right]_{N}^{\xi_{1}} \cdot\left[\vec{x}^{n+1}-\vec{x}^{n}\right]^{\xi_{1}}+\left[\vec{F}_{\mathrm{cont}}^{n+1 / 2}\right]_{N}^{\xi_{2}} \cdot\left[\vec{x}^{n+1}-\vec{x}^{n}\right]^{\xi_{2}} \\
& =t_{N}\left(\vec{x}^{\xi_{1}}, t^{n+1 / 2}\right)\left[g_{d}^{n+1}-g_{d}^{n}\right]=-\left[U\left(g_{d}^{n+1}\right)-U\left(g_{d}^{n}\right)\right] .
\end{aligned}
$$

During the first step between configurations 1 and 2, we have, using Eq. (56), $g_{d}^{1}=g^{1}>0, U\left(g^{1}\right)=$ 0 and $g_{d}^{2} \leqslant 0, U\left(g_{d}^{2}\right) \geqslant 0$, leading to

$$
W_{\text {cont }}^{2}=\underbrace{W_{\text {cont }}^{1}}_{=0}-U\left(g_{d}^{2}\right)+\underbrace{U\left(g_{d}^{1}\right)}_{=0}=-U\left(g_{d}^{2}\right) \leqslant 0 .
$$

During step $n \in\left[2, n^{\prime}\right]$, we have $g_{d}^{n} \leqslant 0, U\left(g_{d}^{n}\right) \geqslant 0$ and $g_{d}^{n+1} \leqslant 0, U\left(g_{d}^{n+1}\right) \geqslant 0$, leading to

$$
W_{\mathrm{cont}}^{n}=\underbrace{W_{\mathrm{cont}}^{n-1}+U\left(g_{d}^{n-1}\right)}_{=0}-U\left(g_{d}^{n}\right)=-U\left(g_{d}^{n}\right)<0 .
$$

Finally, considering the step $n^{\prime}$, we have $g_{d}^{n^{\prime}}>0, U\left(g_{d}^{n^{\prime}}\right)>0$ and $g_{d}^{n^{\prime}+1}>0, U\left(g_{d}^{n^{\prime}+1}\right)=0$, leading to

$$
W_{\text {cont }}^{n^{\prime}+1}=\underbrace{W_{\text {cont }}^{n^{\prime}}+U\left(g_{d}^{n^{\prime}}\right)}_{=0}-U\left(g_{d}^{n^{\prime}+1}\right)=-U\left(g_{d}^{n^{\prime}+1}\right)=0 .
$$

Therefore, the normal part of Eq. (26) is verified: during persistent contact the work is negative, and after the contact is released, the work is equal to zero. Let us note that during this last step where the contact is released, the pressure computed by Eq. (57) is different from zero, and therefore, since $U\left(g_{d}^{n^{\prime}}\right)>0$, the contact forces are different from zero too. 


\subsubsection{Numerical dissipation}

If the time integration scheme used is the EDMC-1 algorithm, Armero and Petöcz [7] have introduced numerical dissipation related to the contact forces with

$$
\begin{aligned}
& {\left[F_{\text {diss }}^{n+1 / 2}\right]^{\xi_{1}}=-\chi \frac{U\left(g_{d}^{n+1}-g_{d}^{n}\right)}{g_{d}^{n+1}-g_{d}^{n}} \vec{n}\left(\vec{x}^{\xi_{1}}, t^{n+1 / 2}\right),} \\
& {\left[\vec{F}_{\text {diss }}^{n+1 / 2}\right]^{\xi_{2}}=\chi \frac{U\left(g_{d}^{n+1}-g_{d}^{n}\right)}{g_{d}^{n+1}-g_{d}^{n}} \varphi^{\xi_{2}}\left(u^{n+1 / 2}\right) \vec{n}\left(\vec{x}^{\xi_{1}}, t^{n+1 / 2}\right),}
\end{aligned}
$$

where $\chi$ is a parameter governing the numerical dissipation. This expression of the dissipation forces verifies directly Eqs. (28). Moreover, one gets

$$
\sum_{i=1,2}\left[\vec{F}_{\text {diss }}^{n+1 / 2}\right]^{\xi_{i}} \cdot\left[\vec{x}^{n+1}-\vec{x}^{n}\right]^{\xi_{i}}=\chi U\left(g_{d}^{n+1}-g_{d}^{n}\right)=\Delta_{F} \geqslant 0,
$$

which verifies Eq. (29).

\subsection{Friction forces}

Let us assume that the projection of the slave node in the mid-configuration is $\vec{y}^{n+1 / 2}\left(\right.$ no, $\left.u^{c n+1 / 2}\right)$. Our first simplification is to define an orthogonal unit base associated to this point:

$$
\begin{aligned}
& \vec{t}_{1}^{c}\left(\vec{x}^{\xi_{1}}, t^{n+1 / 2}\right)=\frac{\vec{n}^{c}\left(\vec{x}^{\xi_{1}}, t^{n+1 / 2}\right) \wedge\left[\vec{y}^{n+1 / 2}\left(\overline{n o}, \bar{u}_{d}\right)-\vec{y}^{n+1 / 2}\left(n o, u^{c n+1 / 2}\right)\right]}{\left\|\vec{n}^{c}\left(\vec{x}^{\xi_{1}}, t^{n+1 / 2}\right) \wedge\left[\vec{y}^{n+1 / 2}\left(\overline{n o}, \bar{u}_{d}\right)-\vec{y}^{n+1 / 2}\left(n o, u^{c n+1 / 2}\right)\right]\right\|} \wedge \vec{n}^{c}\left(\vec{x}^{\xi_{1}}, t^{n+1 / 2}\right), \\
& \vec{t}_{2}^{c}\left(\vec{x}^{\xi_{1}}, t^{n+1 / 2}\right)=\vec{t}_{1}^{c}\left(\vec{x}^{\xi_{1}}, t^{n+1 / 2}\right) \wedge \vec{n}^{c}\left(\vec{x}^{\xi_{1}}, t^{n+1 / 2}\right) .
\end{aligned}
$$

Therefore the dual base defined by Eq. (41) is equivalent to the primal base.

To integrate Eq. (39) in time, we first compute a dynamic tangential gap increment

$$
\Delta \vec{g}_{t_{d}} \equiv\left[\vec{x}^{n+1}\right]^{\xi_{1}}-\left[\vec{x}^{n}\right]^{\xi_{1}}-\vec{y}^{n+1}\left(n o, u^{c n+1 / 2}\right)+\vec{y}^{n}\left(n o, u^{c n+1 / 2}\right) .
$$

Let us define a dynamical projection, denoted by $\vec{y}\left(n o_{d}, u_{d}\right)$, that corresponds to the accumulation of the dynamic tangential gap increments. This dynamical projection is evaluated in the following way. Firstly, $n o_{d}$ and $u_{d}$ are initialized at the first contact configuration. Next, the dynamic tangent gap is evaluated from the dynamic tangential gap increments and from the difference between the previous dynamical projection (i.e. $\left.\vec{y}^{n+1 / 2}\left(n o_{d}^{n}, u_{d}^{n}\right)\right)$ and the actual projection (i.e. $\left.\vec{y}^{n+1 / 2}\left(n o, u^{c n+1 / 2}\right)\right)$. It becomes

$$
\vec{g}_{t_{d}}=\Delta \vec{g}_{t_{d}}+\left[\vec{y}^{n+1 / 2}\left(n o_{d}^{n}, u_{d}^{n}\right)-\vec{y}^{n+1 / 2}\left(n o, u^{c n+1 / 2}\right)\right] .
$$

As can be seen in Fig. 5, when the dynamic gap is added to the actual projection (i.e. $\left.\vec{y}^{n+1 / 2}\left(n o, u^{c n+1 / 2}\right)\right)$, it does not lead to a point that is on the master surface $\mathbb{S}^{2}$. Therefore, by analogy with Eq. (39), we project this point in the plane tangent to the master surface, leading to point $\overrightarrow{\tilde{x}}$ defined by

$$
\overrightarrow{\tilde{x}}=\vec{y}^{n+1 / 2}\left(n o, u^{c n+1 / 2}\right)+\left[\vec{t}_{\alpha}^{c}\left(\vec{x}^{\xi_{1}}, t^{n+1 / 2}\right) \cdot \vec{g}_{t_{d}}\right]_{\alpha}^{c}\left(\vec{x}^{\xi_{1}}, t^{n+1 / 2}\right) .
$$




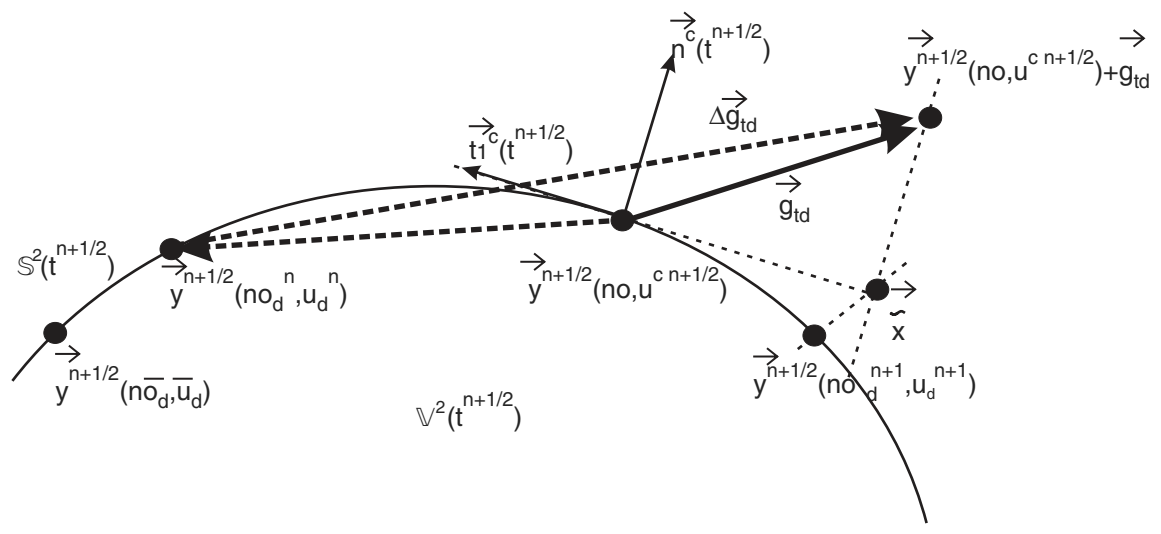

Fig. 5. Construction to find the dynamical projection (2 dimensions).

Actually, the new dynamic values $n o_{d}^{n+1}$ and $u_{d}^{n+1}$ are obtained by projecting $\tilde{x}$ on the surface $\mathbb{S}^{2}$ in the configuration $n+\frac{1}{2}$, leading to $\vec{y}^{n+1 / 2}\left(n o_{d}^{n+1}, u_{d}^{n+1}\right)$.

This construction is illustrated in Fig. 5 and is a planar approximation of a time discretization of Eq. (39) as we will see. If $\tilde{u}$ are the surface coordinates in the new base, and assuming that the gap remains small enough, Eq. (39) becomes

$$
\left[\dot{\vec{x}}^{\xi_{1}}-\varphi_{\delta}^{\xi_{2}} \dot{\vec{x}}^{\xi_{2}}\right] \cdot \vec{t}_{\alpha}=A_{\alpha \beta} \dot{\tilde{u}}_{\beta} \quad \text { with } A_{\alpha \beta}=\vec{t}_{\beta} \cdot \vec{t}_{\alpha}=\delta_{\alpha \beta} .
$$

For a planar approximation, we have $\overrightarrow{\tilde{x}}=\vec{y}^{n+1 / 2}\left(n o_{d}^{n+1}, u_{d}^{n+1}\right)$ and using the definition of $\tilde{u}$, it yields

$$
\begin{aligned}
& \vec{y}^{n+1 / 2}\left(n o_{d}^{n}, u_{d}^{n}\right)-\vec{y}^{n+1 / 2}\left(n o, u^{c n+1 / 2}\right)=\left[\tilde{u}_{d}^{n}\right]_{\alpha} \vec{t}_{\alpha}^{c}\left(\vec{x}^{\xi_{1}}, t^{n+1 / 2}\right), \\
& \vec{y}^{n+1 / 2}\left(n o_{d}^{n+1}, u_{d}^{n+1}\right)-\vec{y}^{n+1 / 2}\left(n o, u^{c n+1 / 2}\right)=\left[\tilde{u}_{d}^{n+1}\right]_{\alpha} \vec{t}_{\alpha}^{c}\left(\vec{x}^{\xi_{1}}, t^{n+1 / 2}\right) .
\end{aligned}
$$

These expressions reduce Eqs. (68-70) to

$$
\begin{aligned}
{\left[\tilde{u}_{d}^{n+1}-\tilde{u}_{d}^{n}\right]_{\alpha}=} & \vec{t}_{\alpha}^{c}\left(\vec{x}^{\xi_{1}}, t^{n+1 / 2}\right) \cdot\left[\vec{x}^{n+1}-\vec{x}^{n}\right]^{\xi_{1}} \\
& -\vec{t}_{\alpha}^{c}\left(\vec{x}^{\xi_{1}}, t^{n+1 / 2}\right) \cdot\left[\vec{y}^{n+1}\left(n o, u^{c n+1 / 2}\right)-\vec{y}^{n}\left(n o, u^{c n+1 / 2}\right)\right],
\end{aligned}
$$

which is a time integration of Eq. (71).

If the master surface is not plane, these approximations consist in evaluating the tangential gap by joining the projections by a line and not by moving on the surface. But this approximation is of the same order as the finite element decomposition that leads to bilinear Coons patches.

Now we have to evaluate the frictional forces from these dynamic coordinates. Let us assume that the sticking point is on the entity $\bar{n}$ and has the coordinates $\bar{u}_{d}$. Therefore, the sticking predictor is evaluated as

$$
t_{T_{\alpha}}^{\mathrm{pred}}\left(x^{\xi_{1}}, t^{n+1 / 2}\right)=-k_{T} r_{c}\left[\frac{\vec{y}^{n+1 / 2}\left(n o_{d}^{n}, u_{d}^{n}\right)+\vec{y}^{n+1 / 2}\left(n o_{d}^{n+1}, u_{d}^{n+1}\right)}{2}-\vec{y}^{n+1 / 2}\left(n \bar{o}, \bar{u}_{d}\right)\right] \cdot \vec{t}_{\alpha}^{c}\left(\vec{x}^{\xi_{1}}, t^{n+1 / 2}\right)
$$

with $k_{T}$ the tangential penalty and $r_{c}$ a factor that ensures an increase of the master surface size will not introduce numerical energy in the system. This ratio will be computed when evaluating 
the friction dissipation (Section 4.2.3). Using the Coulomb law defined by Eq. (43), the final components are

$$
t_{T_{\alpha}}= \begin{cases}t_{T_{\alpha}}^{\text {pred }} & \text { if } \Phi_{c}\left(t_{T_{\alpha}}^{\text {pred }}\right) \leqslant 0, \\ \frac{t_{T_{\alpha}}^{\text {pred }}\left(\vec{x}^{\xi_{1}}, t^{n+1 / 2}\right)}{\sqrt{t_{T_{\beta}}^{\text {pred }} t_{T_{\beta}}^{\text {pred }}}} \mu_{c} t_{N}\left(\vec{x}^{\xi_{1}}, t^{n+1 / 2}\right) & \text { if } \Phi_{c}\left(t_{T_{\alpha}}^{\text {pred }}\right)>0 .\end{cases}
$$

Since the dual base corresponds to the new base, assuming the normal gap remains small, the frictional forces from Eq. (47) becomes

$$
\begin{aligned}
& {\left[\vec{F}_{\text {cont }}^{n+1 / 2}\right]_{T}^{\xi_{1}}=t_{T_{\alpha}}\left(\vec{x}^{\xi_{1}}, t^{n+1 / 2}\right) \vec{t}_{\alpha}^{c}\left(\vec{x}^{\xi_{1}}, t^{n+1 / 2}\right),} \\
& {\left[\vec{F}_{\text {cont }}^{n}\right]_{T}^{\xi_{2}}=-t_{T_{\alpha}}\left(\vec{x}^{\xi_{1}}, t^{n+1 / 2}\right) \varphi_{\delta}^{\xi_{2}}\left(n o, u^{c n+1 / 2}\right) \vec{t}_{\alpha}^{c}\left(\vec{x}^{\xi_{1}}, t^{n+1 / 2}\right) .}
\end{aligned}
$$

\subsubsection{Conservation of linear momentum}

Since $1-\sum_{\xi_{2}} \varphi^{\xi_{2}}\left(\right.$ no, $\left.u^{c n+1 / 2}\right)=0$, Eq. (20) is directly verified since Eqs. (76) lead to

$$
\left[\vec{F}_{\text {cont }}^{n+1 / 2}\right]_{T}^{\xi_{1}}+\sum_{\xi_{2}}\left[\vec{F}_{\text {cont }}^{n+1 / 2}\right]_{T}^{\xi_{2}}=0 .
$$

\subsubsection{Conservation of angular momentum}

Eqs. (76) lead to

$$
\begin{aligned}
& \sum_{i=1,2}\left[\frac{\vec{x}^{n+1}+\vec{x}^{n}}{2}\right]^{\xi_{i}} \wedge\left[\vec{F}_{\text {cont }}^{n+1 / 2}\right]_{T}^{\xi_{i}} \\
& =t_{T_{\alpha}}\left(\vec{x}^{\xi_{1}}, t^{n+1 / 2}\right) \underbrace{\left[\left[\frac{\vec{x}^{n+1}+\vec{x}^{n}}{2}\right]^{\xi_{1}}-\varphi_{\delta}^{\xi_{2}}\left(n o, u^{c n+1 / 2}\right)\left[\frac{\vec{x}^{n+1}+\vec{x}^{n}}{2}\right]^{\xi_{2}}\right]}_{=g\left(\vec{x}^{\xi_{1}}, t^{n+1 / 2}\right) \vec{n}^{c}\left(\vec{x}^{\xi_{1}}, t^{n+1 / 2}\right)} \wedge \vec{t}_{\alpha}^{c}\left(\vec{x}^{\xi_{1}}, t^{n+1 / 2}\right),
\end{aligned}
$$

which is different from zero since the term depending on the gap $g$ in Eq. (47) was neglected. Eq. (22) is therefore verified only if the gap remains small enough.

\subsubsection{Evaluation of frictional dissipation}

In this section, we will evaluate the scaling factor $r_{c}$ used in Eq. (74) that leads to a consistent algorithm. The tangential part of Eq. (26) is computed from Eq. (76), yielding

$$
\begin{aligned}
\Delta_{\mathrm{fr}} & =-\left[\vec{F}_{\text {cont }}^{n+1 / 2}\right]_{T}^{\xi_{1}} \cdot\left[\vec{x}^{n+1}-\vec{x}\right]^{\xi_{1}}-\left[\vec{F}_{\text {cont }}^{n}\right]_{T}^{\xi_{2}} \cdot\left[\vec{x}^{n+1}-\vec{x}\right]^{\xi_{2}} \\
& =-t_{T_{\alpha}}\left(\vec{x}^{\xi_{1}}, t^{n+1 / 2}\right) \vec{t}_{\alpha}^{c}\left(\vec{x}^{\xi_{1}}, t^{n+1 / 2}\right) \cdot\left\{\left[\vec{x}^{n+1}-\vec{x}^{n}\right]^{\xi_{1}}-\varphi_{\delta}^{\xi_{2}}\left(n o, u^{c n+1 / 2}\right)\left[\vec{x}^{n+1}-\vec{x}^{n}\right]^{\xi_{2}}\right\} .
\end{aligned}
$$

Using Eqs. (68-70), this last relation becomes

$$
\begin{aligned}
\Delta_{\mathrm{fr}} & =-t_{T_{\alpha}}\left(\vec{x}^{\xi_{1}}, t^{n+1 / 2}\right) \vec{t}_{\alpha}^{c}\left(\vec{x}^{\xi_{1}}, t^{n+1 / 2}\right) \cdot \Delta \vec{g}_{t_{d}} \\
& =-t_{T_{\alpha}}\left(\vec{x}^{\xi_{1}}, t^{n+1 / 2}\right) \vec{t}_{\alpha}^{c}\left(\vec{x}^{\xi_{1}}, t^{n+1 / 2}\right) \cdot\left[\overrightarrow{\tilde{x}}^{n+1 / 2}\left(n o_{d}^{n}, u_{d}^{n}\right)\right] \\
& \simeq-t_{T_{\alpha}}\left(\vec{x}^{\xi_{1}}, t^{n+1 / 2}\right) \vec{t}_{\alpha}^{c}\left(\vec{x}^{\xi_{1}}, t^{n+1 / 2}\right) \cdot\left[\vec{y}^{n+1 / 2}\left(n o_{d}^{n+1}, u_{d}^{n+1}\right)-\vec{y}^{n+1 / 2}\left(n o_{d}^{n}, u_{d}^{n}\right)\right] .
\end{aligned}
$$


As Armero and Petöcz proposed [8], let us define $\eta_{d}$ a value that represents the slip but that does not depend on the surface size variation and let us define $\Delta \vec{y}$ such that

$$
\begin{aligned}
& \eta_{d}^{n} \equiv\left[\vec{y}_{0}\left(n o_{d}^{n}, u_{d}^{n}\right)-\vec{y}_{0}\left(n \bar{o}, \bar{u}_{d}\right)\right] \cdot\left[\vec{y}_{0}\left(n o_{d}^{n}, u_{d}^{n}\right)-\vec{y}_{0}\left(n \bar{o}, \bar{u}_{d}\right)\right], \\
& \Delta \vec{y}^{n} \equiv\left[\vec{y}^{n+1 / 2}\left(n o_{d}^{n}, u_{d}^{n}\right)-\vec{y}^{n+1 / 2}\left(n \bar{o}, \bar{u}_{d}\right)\right], \\
& \Delta \vec{y}^{n+1} \equiv\left[\vec{y}^{n+1 / 2}\left(n o_{d}^{n+1}, u_{d}^{n+1}\right)-\vec{y}^{n+1 / 2}\left(n \bar{o}, \bar{u}_{d}\right)\right] .
\end{aligned}
$$

Therefore Eq. (74) leads to

$$
\begin{aligned}
& t_{T_{\alpha}}^{\text {pred }}\left(\vec{x}^{\xi_{1}}, t^{n+1 / 2}\right) \vec{t}_{\alpha}^{c}\left(\vec{x}^{\xi_{1}}, t^{n+1 / 2}\right) \cdot\left[\vec{y}^{n+1 / 2}\left(n o_{d}^{n+1}, u_{d}^{n+1}\right)-\vec{y}^{n+1 / 2}\left(n o_{d}^{n}, u_{d}^{n}\right)\right] \\
& \quad=-\frac{1}{2} k_{T} r_{c} \vec{t}_{\alpha}^{c}\left(\vec{x}^{\xi_{1}}, t^{n+1 / 2}\right) \cdot\left[\Delta \vec{y}^{n}+\Delta \vec{y}^{n+1}\right] \vec{t}_{\alpha}^{c}\left(\vec{x}^{\xi_{1}}, t^{n+1 / 2}\right) \cdot\left[\Delta \vec{y}^{n+1}-\Delta \vec{y}^{n}\right] \\
& \quad=-\frac{k_{T}}{2}\left[\eta_{d}^{n+1}-\eta_{d}^{n}\right]
\end{aligned}
$$

if $r_{c}$ is defined by

$$
r_{c}= \begin{cases}\frac{\eta_{d}^{n+1}-\eta_{d}^{n}}{\Delta^{2} y\left(n o_{d}^{n+1}, u_{d}^{n+1}\right)-\Delta^{2} y\left(n o_{d}^{n}, u_{d}^{n}\right)} & \text { if } n o_{d}^{n+1}, u_{d}^{n+1} \neq n o_{d}^{n}, u_{d}^{n}, \\ \frac{\eta_{d}^{n+1}}{\Delta^{2} y\left(n o_{d}^{n+1}, u_{d}^{n+1}\right)} & \text { if } n o_{d}^{n+1}, u_{d}^{n+1}=n o_{d}^{n}, u_{d}^{n}\end{cases}
$$

with $\Delta^{2} y\left(n o_{d}^{n+1}, u_{d}^{n+1}\right)=\sum_{\alpha=1}^{2}\left\{\Delta \vec{y}^{n+1} \cdot \vec{t}_{\alpha}^{c}\left(\vec{x}^{\xi_{1}}, t^{n+1 / 2}\right)\right\}^{2}$. Therefore, using Eqs. (75) and (82), Eq. (80) can be rewritten as

$$
\Delta_{\mathrm{fr}}= \begin{cases}\frac{k_{T}}{2}\left[\eta_{d}^{n+1}-\eta_{d}^{n}\right] & \text { if } \Phi_{c}\left(\vec{T}^{\mathrm{pred}}\right) \leqslant 0, \\ \frac{k_{T} \mu_{c} t_{N}}{2\left\|\vec{T}^{\mathrm{pred}}\right\|}\left[\eta_{d}^{n+1}-\eta_{d}^{n}\right] & \text { if } \Phi_{c}\left(\vec{T}^{\mathrm{pred}}\right)>0 .\end{cases}
$$

Now we have to verify the physical consistency of this relation and to determine the sticking point. Let us define a cycle of $n^{\prime}$ steps without sliding (i.e. sticking status) and the following step with a sliding status. Let us suppose that the contact starts at step 0 . In such a case, the sticking point is defined by $\bar{n}=n o_{d}^{0}$ and by $\bar{u}=u_{d}^{0}$. Thanks to Eq. (81) it leads to $\eta_{d}^{0}=0$ and Eq. (84) leads to

$$
\begin{aligned}
\Delta_{\mathrm{fr}} & =\sum_{n=0}^{n=n^{\prime}-1} \frac{k_{T}}{2}\left[\eta_{d}^{n+1}-\eta_{d}^{n}\right]+\frac{k_{T} \mu_{c} t_{N}}{2\left\|\vec{T}^{\text {pred }}\right\|}\left[\eta_{d}^{n^{\prime}+1}-\eta_{d}^{n^{\prime}}\right] \\
& =\frac{k_{T}}{2} \eta_{d}^{n^{\prime}}\left[1-\frac{\mu_{c} t_{N}}{\left\|\vec{T}^{\text {pred }}\right\|}\right]+\frac{k_{T} \mu_{c} t_{N}}{2\left\|\vec{T}^{\text {pred }}\right\|} \eta_{d}^{n^{\prime}+1}>0 .
\end{aligned}
$$

Here we do the same approximation that Armero and Petöcz did by assuming the new sticking point is the latest obtained projection point: $\bar{u}_{d}^{n^{\prime}+1}=u_{d}^{n^{\prime}+1}$. This approximation leads to the exact 
solution when the tangential penalty tends to infinity. For such a penalty, we have $u_{d}^{n^{\prime}}=\bar{u}$ and, using Eqs. (75) and (81), Eq. (85) is rewritten as

$$
\begin{aligned}
\Delta_{\mathrm{fr}} & =\frac{k_{T} \mu_{c} t_{N}}{2\left\|\vec{T}^{\text {pred }}\right\|} \eta_{d}^{n^{\prime}+1}=\frac{k_{T} \mu_{c} t_{N}}{2\left\|\vec{T}^{\text {pred }}\right\|}\left\|\vec{y}_{0}\left(n \bar{o}_{d}^{n^{\prime}+1}, \bar{u}_{d}^{n^{\prime}+1}\right)-\vec{y}_{0}\left(\overline{n o}, \bar{u}_{d}\right)\right\|^{2} \\
& =\mu_{c} t_{N}\left\|\vec{y}_{0}\left(\overline{n o}_{d}^{n^{\prime}+1}, \bar{u}_{d}^{n^{\prime}+1}\right)-\vec{y}_{0}\left(\overline{n o}, \bar{u}_{d}\right)\right\|,
\end{aligned}
$$

which corresponds to the time integration of Eq. (48). If the penalty is different from infinity (which is always the case), the energy stored in $\left(k_{T} / 2\right) \eta_{d}^{n^{\prime}}\left[1-\mu_{c} t_{N} /\left\|\vec{T}^{\text {pred }}\right\|\right]$ is lost and the scheme overestimates the dissipation.

Since the tangent forces are physically dissipative, we do not need to define additional dissipation forces related to these tangent contact forces.

\section{Numerical examples}

In this section we will prove that our method to compute the contact interactions is both efficient and robust. The elements are 8-node bricks with underintegration of the pressure to avoid volumic locking. The model used is an elasto-plastic hypoelastic material. The internal forces expression can be found in [21,22]. Normal contact forces are expressed by Eq. (58) and tangent contact forces are expressed by Eq. (76). Expression of the dissipation forces related to the internal forces and expression of the dissipation velocities corresponding to a first-order dissipation algorithm (EDMC-1) can be found in [25]. Dissipation forces related to the normal contact forces are expressed by Eq. (65).

\subsection{Numerical example 1: impact of two hollow cylinders}

The problem under consideration is the interaction of two hollow perpendicular cylinders (Fig. 6a). Both cylinders have the same mean radius $(R=98.5 \mathrm{~mm})$, the same thickness $(e=3 \mathrm{~mm})$ and the same length $(L=460 \mathrm{~mm})$. The initial difference of the gravity center coordinates is $\vec{x}=(250 \mathrm{~mm} ; 0 \mathrm{~mm} ; 0 \mathrm{~mm})$. The right cylinder has no initial velocity, while the left one has an initial velocity $\dot{\vec{x}}=(40 \mathrm{~m} / \mathrm{s} ; 4 \mathrm{~m} / \mathrm{s} ; 0 \mathrm{~m} / \mathrm{s})$. Both cylinders are made of aluminum (density $\rho=2710 \mathrm{~kg} / \mathrm{m}^{3}$, Young's modulus $Y=70000 \mathrm{~N} / \mathrm{mm}^{2}$, Poisson's ratio $v=0.3$, initial yield stress $\Sigma_{0}=90 \mathrm{~N} / \mathrm{mm}^{2}$ and linear isotropic hardening $h=100 \mathrm{~N} / \mathrm{mm}^{2}$ ). Each cylinder has 990 elements ( 3 through the thickness, 22 along the circumference, 15 along the length). The interaction between the cylinders occurs with a Coulomb frictional law (normal penalty $k_{N}=10^{5}$, tangential penalty $k_{T}=10^{3}$, friction coefficient $\mu_{c}=0.1$ ). We will compare the results obtained with the following algorithms:

- EDMC-1 algorithm with an infinity spectral radius equal to 0.8 ;

- Hilber-Hughes-Taylor (HHT) [28], with an infinity spectral radius equal to 0.8 .

Fig. $6 \mathrm{~b}$ and c illustrate respectively the equivalent plastic deformations obtained for the EDMC-1 scheme and for the HHT scheme for a time step equal to $0.5 \mu$ s. Both simulations lead to the same 


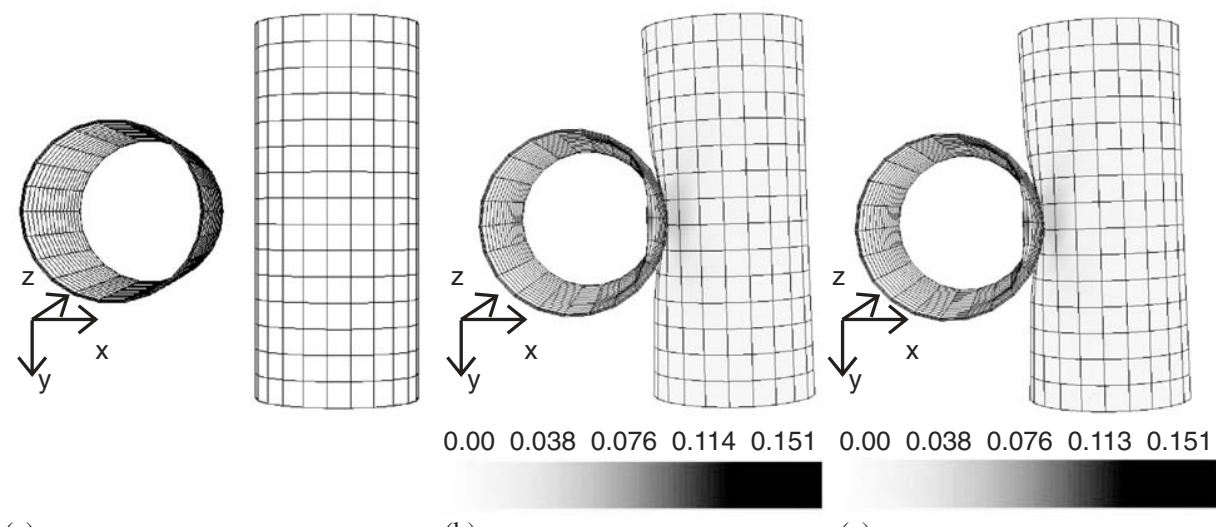

(a)

(b)

(c)

Fig. 6. Geometry of the two cylinders: (a) initial configuration; (b) equivalent plastic deformation (after $5 \mathrm{~ms}$ ) with the EDMC-1 simulation; (c) equivalent plastic deformation (after $5 \mathrm{~ms}$ ) with the HHT simulation.

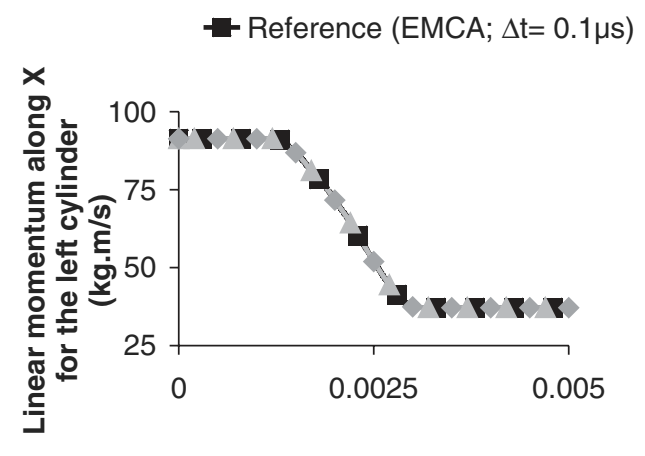

(a)

Time (s)



(b)

Time (s)

Fig. 7. Time evolution of the left cylinder linear momentum for a time step size equal to $5 \mu$ s: (a) linear momentum along $X$; (b) linear momentum along $Y$.

results. Fig. 7a and $\mathrm{b}$ illustrate the time evolution of the left cylinder linear momentum, respectively along axis $X$ and $Y$. The solutions are compared to a reference solution obtained with the EDCM scheme without numerical dissipation (EMCA) and a time step equal to $0.1 \mu$ s. Along direction $X$, the left cylinder transmits a part of its linear momentum to the right cylinder. Along direction $Y$, the friction interaction leads to a transfer of linear momentum from the left cylinder to the right cylinder. All the solutions are identical. Fig. 8a illustrates the fact that, since the left cylinder impacts the right cylinder below its gravity center (positive $Y$ ), the angular momentum along $Z$ of the left cylinder begins to increase. Next, due to the friction effects, the angular momentum along $Z$ of the left cylinder decreases. The EDMC-1 scheme leads to a solution different by about $10 \%$ from the reference solution. The HHT scheme leads to a solution different by about $35 \%$ to the reference solution. The angular momentum along $Z$ of the two cylinders is illustrated in Fig. 8b. Since we have neglected the term depending on the gap $g$ in Eq. (47), the 


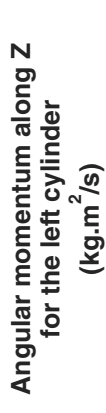

(a)
Reference (EMCA; $\Delta \mathrm{t}=0.1 \mu \mathrm{s})$

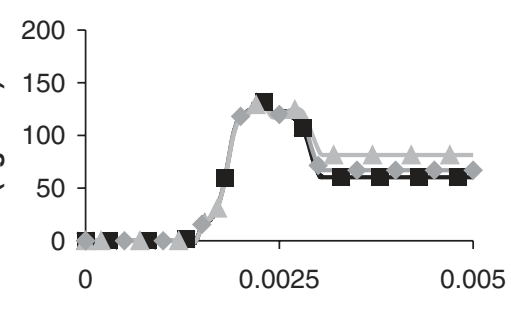

Time (s) (b)

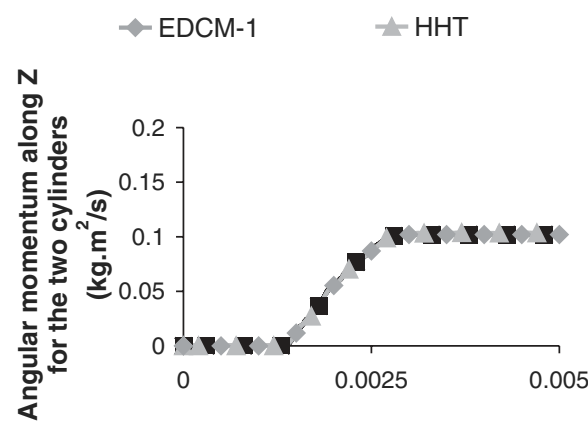

$\leadsto$ EDCM-1 $\quad$ HHT

Time (s)

Fig. 8. Time evolution of the angular momentum for a time step size equal to $5 \mu$ s: (a) angular momentum along $Z$ for the left cylinder; (b) angular momentum for the two cylinder.

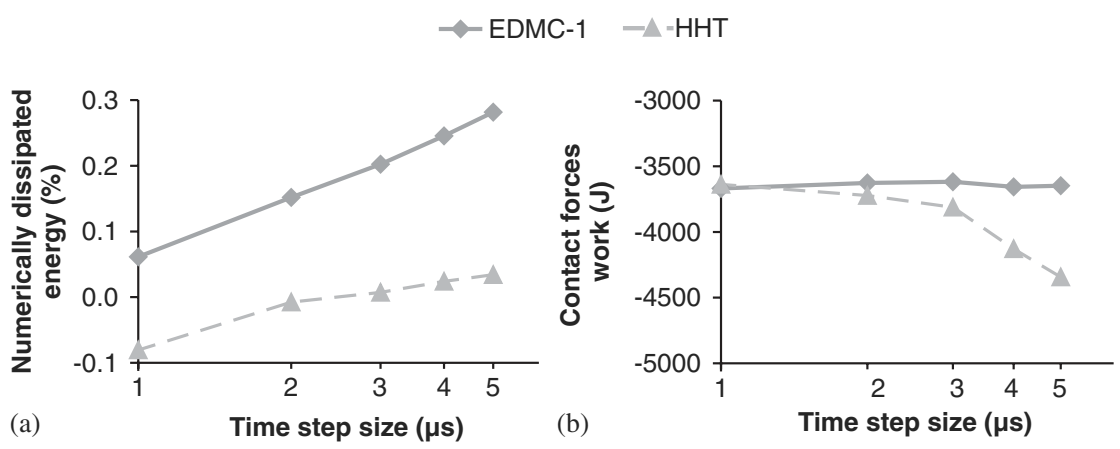

Fig. 9. Final energy (after $5 \mathrm{~ms}$ ) of the two cylinders: (a) numerically dissipated energy; (b) contact forces work.

value is not constant but has a variation of $0.1 \%$. Fig. 9a illustrates the numerically dissipated energy at the end of the simulation. The energy dissipative simulations are performed with 5 constant time step sizes: 1, 2, 3, 4 and $5 \mu$ s. For small time steps size, the HHT algorithm introduces energy in the system and is dissipative only for large time step sizes. The EDMC-1 algorithm, whatever the time step size, always leads to a positive numerical dissipation. Fig. 9b illustrates the (accumulated) work of the contact forces at the end of the simulation. For the EDMC-1 scheme, this work corresponds to the friction dissipation and this value is constant for each time step size. For the HHT scheme, the normal component does not lead to a work equal to zero. Therefore, the work of contact force is not always equal to the friction dissipation and can vary by about $20 \%$ when the time step size is multiplied by 5 .

\subsection{Numerical example 2: buckling of square tubes}

Let us now study the dynamic buckling of a square tube as proposed by Karagiozova and Jones [29]. Due to symmetry, only one quarter of the tube is under consideration. The square tube (Fig. 10a) has a constant thickness of $1.14 \mathrm{~mm}$ and a height of $146 \mathrm{~mm}$. The cross square section of the tube has a length of $23.7 \mathrm{~mm}$, and the angles are smoothed with a corner radius of $2.1 \mathrm{~mm}$. If 

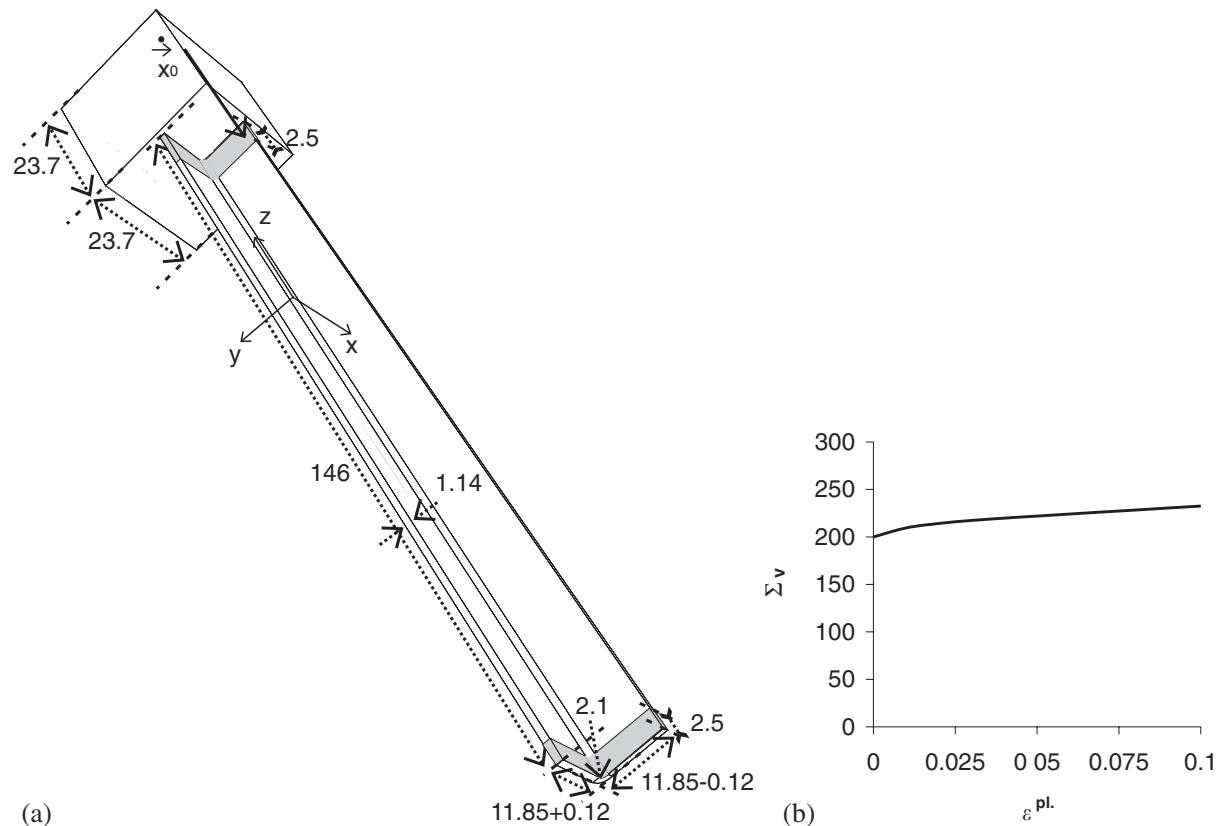

Fig. 10. Description of the square tube: (a) geometry (mm); (b) hardening law (MPa).

the section is kept symmetric, the dynamic buckling will be different from the experimentation that is not perfectly symmetric, leading to a non-symmetrical buckling. Therefore Karagiozova and Jones [29] have proposed to reduce the $Y$-length of the section by $1 \%$ and to increase the $X$ length of the section by $1 \%$ (Fig. 10a) to introduce some numerical imperfections in the model. Therefore, the area of the section remains constant with a little asymmetry. The tube is made of aluminum (density $\rho=2700 \mathrm{~kg} / \mathrm{m}^{3}$, Young's modulus $Y=71000 \mathrm{~N} / \mathrm{mm}^{2}$ and Poisson's ratio $v=0.3)$. The von Mises limit $\Sigma_{v}$ depends on the equivalent plastic strain $\varepsilon^{p l}$ through the law $\Sigma_{v}(\mathrm{MPa})=200+11.64\left[1-\mathrm{e}^{-100 \varepsilon^{p l}}\right]+209 \varepsilon^{p l}(\mathrm{MPa})$. This hardening law is illustrated in Fig. $10 \mathrm{~b}$. Let us note that Karagiozova and Jones [29] use a multi-linear-segment approximation of this law. Our numerical model is composed of 2600 brick elements (120 along the height, 10 along each half length of the section, 2 along the arc and 1 through the thickness).

The buckling is generated thanks to the impact of a rigid mass $M$, with an initial velocity $\dot{\vec{x}}_{0}$ (Fig. 10a). The rigid mass is simulated with a volume of length $23.7 \mathrm{~mm}$ and with a Young's modulus ten times larger than the tube's one. When studying the buckling of a tube, with shells elements, Langseth et al. [30], who used shell elements, fixed the rotational degrees of freedom at the extremity of the tube. Since we use brick elements, we do not have this opportunity. Therefore, we choose to fix the radial displacements of the nodes situated at $2.5 \mathrm{~mm}$ of the extremities (gray zones represented in Fig. 10a). The study proposed by Karagiozova and Jones [29] consists in the absorption of an impact of $600 \mathrm{~J}$ (for the whole tube) at different velocities. Values of the impact velocities $\dot{\vec{x}}_{0}$ and of the impact masses $M$ are reported in Table 1 for the four impact simulations under consideration.

We use the EDMC-1 scheme with $\rho_{\infty}=0.8$ (spectral radius at infinity) to simulate the problem. The time step size is computed thanks to an automatic criterion [31] with an accuracy of $10^{-4}$ on 
Table 1

Impact properties

\begin{tabular}{llllll}
\hline Name & $\begin{array}{l}\text { Impact } \\
\text { velocity } \\
(\mathrm{m} / \mathrm{s})\end{array}$ & $\begin{array}{l}\text { Impact } \\
\text { mass, } M \\
(\mathrm{~kg})\end{array}$ & $\begin{array}{l}\text { Experimental } \\
\text { crushing [33] } \\
(\mathrm{mm})\end{array}$ & $\begin{array}{l}\text { Numerical } \\
\text { crushing } \\
(\mathrm{mm})\end{array}$ & $\begin{array}{l}\text { Karagiozova } \\
\text { and Jones [29] } \\
(\mathrm{mm})\end{array}$ \\
\hline N76 & 14.84 & 5.45 & 68.3 & 71.9 & 66.4 \\
G5 & 25.34 & 1.87 & 60.8 & 70 & 54.3 \\
N41 & 64.62 & 0.28 & 44.1 & 46.9 & 39.4 \\
N61 & 98.27 & 0.126 & 35.5 & 38.8 & 35.6 \\
\hline
\end{tabular}

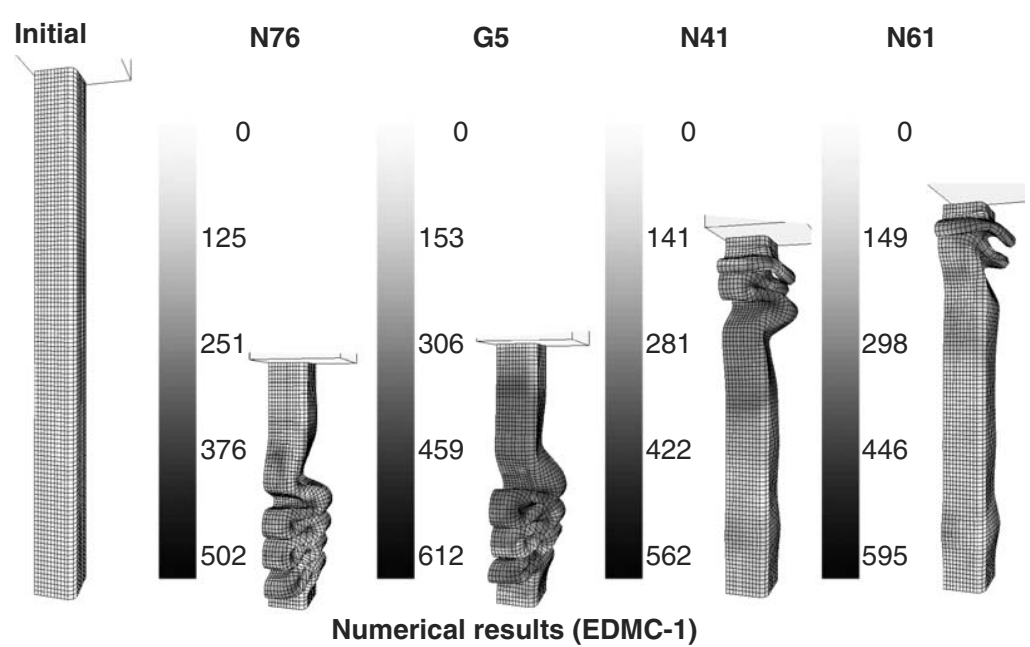

Fig. 11. Final deformations of the square tube: von Mises stress (MPa) of the numerical results obtained with the EDMC-1 algorithm.

the integration error [31] and the choice of updating the Hessian matrix is also computed from automatic criteria [31]. Each time step is computed with a Newton-Raphson scheme (tolerance $10^{-5}$ ) enhanced by a line-search [32] (tolerance $10^{-3}$ ). Contact forces are computed with the formulation developed in Section 4. The contact is frictionless and uses a normal penalty $k_{N}=10^{3}$ for the tubes N61, N41 and G5, but a penalty of $k_{N}=10^{4}$ for the N76 tube that has a slower dynamics.

The deformed configurations obtained are illustrated in Fig. 11. When compared to the experimental results obtained by Yang [33] (Fig. 12), it appears that the buckling modes are quite similar (same number of buckles and same positions). Crushing distances (of the top of the tube) of our numerical results and of the experimental results are reported in Table 1. We have also reported, in this table, the crushing distances obtained with an explicit algorithm and shell elements by Karagiozova and Jones [29]. It appears that our numerical results obtained with an implicit algorithms are accurate with respect to experimental results, with a $15 \%$ error. Moreover, let us note that an implicit simulation with an $\alpha$-generalized algorithm [34] $\left(\rho_{\infty}=0.2\right)$ did not succeed for the present simulation (no convergence of the Newton-Raphson algorithm). 




(a) - Experimental results

Fig. 12. Final deformations of the square tube: experimental results obtained by Yang [33] and published by Jones and Karagiozova [29] (Reprinted from Int J Impact Eng 30, D. Karagiozova and N. Jones, Dynamic buckling of elastic-plastic square tubes under axial impact-II: structural response, 167-92, Copyright 2004, with permission from Elsevier).

\section{Conclusions}

In this paper we have presented a practical way to simulate complex contact interactions with an implicit scheme in the framework of the so-called "conserving algorithm". To achieve this, the evolution of the normal was smoothed and rendered continuous even for facetted discretizations. Next, the contact forces are computed in a thermodynamically consistent way, taking into account this smoothing method. Numerical examples have proved the efficiency and accuracy of this method for impact simulations. We conclude that, with such improvements, implicit schemes are able to simulate complex contact problems.

\section{References}

[1] Laursen T. Computational contact and impact mechanics. Berlin: Springer; 2002.

[2] Wriggers P. Computational contact mechanics. New York: Wiley; 2002.

[3] Belytschko T, Daniel W, Ventura G. A monolithic smoothing-gap algorithm for contact-impact based on the signed distance function. Int J Numer Methods Eng 2002;55:101-25.

[4] Puso M, Laursen T. A mortar segment-to-segment contact method for large deformation solid mechanics. Comput Methods Appl Mech Eng 2004;193:601-27.

[5] Graillet D, Ponthot J-P. Efficient implicit schemes for the treatment of the contact between deformable bodies: applications to shock-absorber devices. Int J Crashworthiness 1999;4(3):173-286.

[6] Simo J, Tarnow N. The discrete energy-momentum method. Conserving algorithms for nonlinear elastodynamics. ZAMP 1992;43:757-92.

[7] Armero F, Petöcz E. Formulation and analysis of conserving algorithms for frictionless dynamic contact/impact problems. Comput Methods Appl Mech Eng 1998;158:269-300.

[8] Armero F, Petöcz E. A new dissipative time-stepping algorithm for frictional contact problems: formulation and analysis. Comput Methods Appl Mech Eng 1999;179:151-78. 
[9] Laursen T, Chawla V. Design of energy conserving algorithms for frictionless dynamic contact problems. Int J Numer Methods Eng 1997;40:863-86.

[10] Chawla V, Laursen T. Energy consistent algorithms for frictional contact problems. Int J Numer Methods Eng 1998;42:799-827.

[11] Laursen T, Love G. Improved implicit integrators for transient impact problems - geometric admissibility within the conserving framework. Int J Numer Methods Eng 2002;53:245-74.

[12] Love G, Laursen T. Improved implicit integrators for transient impact problems - dynamic frictional dissipation within the admissible conserving framework. Comput Methods Appl Mech Eng 2003;192:2223-48.

[13] Noels L. Contributions aux algorithmes d'intégration temporelle conservant l' énergie en dynamique non-linéaire des structures. PhD thesis, University of Liège, 2004 [in French].

[14] Noels L, Stainier L, Ponthot J-P. Combined implicit/explicit algorithms for crashworthiness analysis. Int J Impact Eng 2004;30:1161-77.

[15] Noels L, Stainier L, Ponthot J-P. Energy conserving balance of explicit steps to combine implicit and explicit algorithms in structural dynamics. Comput Methods Appl Mech Eng 2005, accepted for publication.

[16] Hughes T. The finite element method. Englewood Cliffs, NJ: Prentice-Hall; 1987.

[17] Belytschko T, Liu W, Moran B. Nonlinear finite elements for continua and structures. New York: Wiley; 2000.

[18] Antman S. Non linear problems of elasticity. Berlin: Springer; 1995.

[19] Gonzalez O. Exact energy and momentum conserving algorithms for general models in nonlinear elasticity. Comput Methods Appl Mech Eng 2000;190:1763-83.

[20] Meng X, Laursen T. Energy consistent algorithms for dynamic finite deformation plasticity. Comput Methods Appl Mech Eng 2001;191:1639-75.

[21] Noels L, Stainier L, Ponthot J-P. Energy-momentum conserving algorithm for non-linear hypoelastic constitutive models. Int J Numer Methods Eng 2004;59:83-114.

[22] Noels L, Stainier L, Ponthot J-P. On the use of large time steps with an energy-momentum conserving algorithm for non-linear hypoelastic constitutive models. Int J Solids Struct 2004;41:663-93.

[23] Armero F, Romero I. On the formulation of high-frequency dissipative time-stepping algorithms for non-linear dynamics. Part I: low-order methods for two model problems and nonlinear elastodynamics. Comput Methods Appl Mech Eng 2001;190:2603-49.

[24] Armero F, Romero I. On the formulation of high-frequency dissipative time-stepping algorithms for non-linear dynamics. Part II: second-order methods. Comput Methods Appl Mech Eng 2001;190:6783-824.

[25] Noels L, Stainier L, Ponthot J-P. Simulation of complex impact problems with implicit time algorithm: application to a blade-loss problem. Int J Impact Eng, submitted for publication.

[26] Ponthot J-P. Unified stress update algorithms for the numerical simulation of large deformation elasto-plastic and elasto-viscoplastic processes. Int J Plast 2002;18:91-126.

[27] Laursen T, Simo J. A continuum-based finite element formulation for the implicit solution of multibody, large deformation frictional contact problems. Int J Numer Methods Eng 1993;36:3451-85.

[28] Hilber H, Hughes T, Taylor R. Improved numerical dissipation for time integration algorithms in structural dynamics. Earthquake Eng Struct Dynam 1977;5:283-92.

[29] Karagiozova D, Jones N. Dynamic buckling of elastic-plastic square tubes under axial impact-II: structural response. Int J Impact Eng 2004;30:167-92.

[30] Langseth M, Hopperstad O, Berstad T. Crashworthiness of aluminium extrusions: validation of numerical simulation, effect of mass ratio and impact velocity. Int J Impact Eng 1999;22:829-54.

[31] Noels L, Stainier L, Ponthot J-P, Bonini J. Automatic time stepping algorithms for implicit numerical simulations of non-linear dynamics. Adv Eng Software 2002;33(10):581-95.

[32] Crisfield M. Non-linear finite element analysis of solids and structures, vol. 1. New York: Wiley; 2001.

[33] Yang C-C. Dynamic buckling of square tubes. PhD thesis, University of Liverpool, 1997.

[34] Chung J, Hulbert G. A time integration algorithms for structural dynamics with improved numerical dissipations: the generalized- $\alpha$ method. J Appl Mech 1993;60:371-5. 